\title{
WAVE-BREAKING AND PEAKONS FOR A MODIFIED CAMASSA-HOLM EQUATION
}

\author{
GUILONG GUI, YUE LIU, PETER J. OLVER, AND CHANGZHENG QU
}

\begin{abstract}
In this paper, we investigate the formation of singularities and the existence of peaked traveling-wave solutions for a modified Camassa-Holm equation with cubic nonlinearity. The equation is known to be integrable, and is shown to admit a single peaked soliton and multi-peakon solutions, of a different character than those of the Camassa-Holm equation. Singularities of the solutions can occur only in the form of wave-breaking, and a new wave-breaking mechanism for solutions with certain initial profiles is described in detail.
\end{abstract}

Keywords: Modified Camassa-Holm equation, wave breaking, peakons, integrable systems.

AMS Subject Classification (2000): 35B30, 35G25

\section{INTRODUCTION}

This paper is concerned with the following nonlinear partial differential equation ${ }^{1}$

$$
m_{t}+\left(\left(u^{2}-u_{x}^{2}\right) m\right)_{x}+\gamma u_{x}=0
$$

for the function $u(t, x)$ of time $t$ and a single spatial variable $x$, in which $\gamma$ is a constant, and

$$
m=u-u_{x x}
$$

The equation (1.1) has the form of a modified Camassa-Holm equation with cubic nonlinearity. It was proposed as a new integrable system by Fuchssteiner [23] and Olver and Rosenau [48] by applying the general method of tri-Hamiltonian duality to the bi-Hamiltonian representation of the modified Korteweg-deVries equation. Later, it was obtained by Qiao [50] from the two-dimensional Euler equations, where the variables $u(t, x)$ and $m(t, x)$ represent, respectively, the velocity of the fluid and its potential density. In [51] it was shown that equation (1.1) admits a Lax pair, and hence can be solved by the method of inverse scattering. See Section 2 for details.

Applying the scaling transformation

$$
x \longmapsto \epsilon x, \quad t \longmapsto \epsilon^{-1} t, \quad u \longmapsto \epsilon^{2} u
$$

to equation (1.1) produces

$$
\left(\epsilon^{2} u-u_{x x}\right)_{t}+\left[\left(\epsilon^{2} u^{2}-u_{x}^{2}\right)\left(\epsilon^{2} u-u_{x x}\right)\right]_{x}+\gamma u_{x}=0 .
$$

Expanding

$$
u(t, x)=u_{0}(t, x)+\epsilon u_{1}(t, x)+\epsilon^{2} u_{2}(t, x)+\cdots
$$

in powers of the small parameter $\epsilon$, the leading order term $u_{0}(t, x)$ satisfies

$$
-u_{0, x x t}+\left(u_{0, x}^{2} u_{0, x x}\right)_{x}+\gamma u_{0, x}=0 .
$$

Date: March 26, 2012.

${ }^{1}$ We use subscripts to denote partial derivatives throughout. 
We conclude that $v=u_{0, x}$ satisfies the short pulse equation

$$
v_{x t}=\frac{1}{3}\left(v^{3}\right)_{x x}+\gamma v \text {. }
$$

This equation was derived by Schäfer and Wayne [54] as a model for the propagation of ultra-short light pulses in silica optical fibers, which is also an approximation of nonlinear wave packets in dispersive media in the limit of few cycles on the ultra-short pulse scale [9]. Numerical simulations [9] show that the short pulse equation approximation to Maxwell's equations in the case when the pulse spectrum is not narrowly localized around the carrier frequency is better than that obtained from the nonlinear Schrödinger equation. Well-posedness and wave breaking for the short pulse equation have been studied in [54] and [43], respectively. It was shown in [5] that the short pulse equation and the WKI equation [58]

$$
u_{t}=\left(\frac{u_{x x}}{\left(1+u_{x}^{2}\right)^{\frac{3}{2}}}\right)_{x}
$$

belong to the same hierarchy. The WKI equation is gauge equivalent to the $\mathrm{mKdV}$ equation, while the short pulse equation is gauge equivalent to the sine-Gordon equation [53]. Solitons of the $\mathrm{mKdV}$ equation and the sine-Gordon equation correspond to loop solitons of the WKI equation and the short pulse equation, respectively.

The original Camassa-Holm $(\mathrm{CH})$ equation

$$
m_{t}+u m_{x}+2 u_{x} m+\gamma u_{x}=0,
$$

where $m$ is as above, (1.2), can itself be derived from the Korteweg-deVries equation by tri-Hamiltonian duality. The Camassa-Holm $(\mathrm{CH})$ equation $[6,24]$ (see also $[17,36]$ ) was originally proposed as a model for surface waves, and has been studied extensively in the last twenty years because of its many remarkable properties: infinity of conservation laws and complete integrability $[6,19,24]$, with action angle variables constructed using inverse scattering $[3,4,14,18]$, existence of peaked solitons and multi-peakons $[1,6,7]$, geometric formulations $[8,37,44]$, well-posedness and breaking waves, meaning solutions that remain bounded while their slope becomes unbounded in finite time $[10,11,12,13,40]$. Note that the nonlinearity in the $\mathrm{CH}$ equation is quadratic. Two integrable $\mathrm{CH}$-type equations with cubic nonlinearity have been discovered: One is the equation (1.1), and the second is the Novikov equation [45]

$$
m_{t}+u^{2} m_{x}+\frac{3}{2}\left(u^{2}\right)_{x} m=0 .
$$

The integrability, peaked solitons, well-posedness and blow up phenomena to the Novikov equation have been studied extensively [34, 35, 45, 56, 57]. An alternative modified $\mathrm{CH}$ equation was introduced in [26]. Multi-component versions of the $\mathrm{CH}$ equation have been introduced and studied in [15, 21, 27, 31, 32, 33, 48].

Dual integrable nonlinear systems, such as the $\mathrm{CH}$ and modified $\mathrm{CH}$ equations, are endowed with nonlinear dispersion, which, in most cases, enables them to support non-smooth soliton-like structures. A number of additional examples of dual integrable systems derived using the method of tri-Hamiltonian duality can be found in [20, 48]. Dual equations support a remarkable variety of non-analytic solutions [42], including peakons, compactons, tipons, rampons, mesaons, and so on. Convergence of analytic solutions to non-analytic compactons and peakons was proved in $[39,41]$.

The aim of this paper is to investigate whether the modified $\mathrm{CH}$ equation (1.1) has similar remarkable properties as the $\mathrm{CH}$ equation (1.5). Since the modified $\mathrm{CH}$ equation has a cubic nonlinearity which the $\mathrm{CH}$ equation is only quadratic, one expects that the modified $\mathrm{CH}$ equation should also have peaked solitons and wave-breaking. The following two sections review the bi-Hamiltonian structure and geometric formulations of the modified 
$\mathrm{CH}$ equation. In Section 4, detailed blow-up criteria for strong solutions are established; see Theorem 4.2. It is shown that the solutions of the modified $\mathrm{CH}$ equation can only have singularities which correspond to wave breaking. A sufficient condition for wave breaking of strong solutions in finite time is specified in Section 5; see Theorem 5.2. Finally, the existence of single peakon and multi-peakon solutions is demonstrated in Section 6. Interestingly, in contrast to the $\mathrm{CH}$ equation, with $\gamma=0$, we find that multi-peakon solutions of (1.1) have only constant amplitudes.

\section{GEOMETRIC FORMULATION AND INTEGRABILITY}

The $\mathrm{CH}$ equation (1.5) has the three distinct geometric formulations. First, it describes the geodesic flows, respectively, on the Bott-Virasoro group for the case $\gamma \neq 0[44,55]$ and on the diffeomorphism group of the unit circle under $H^{1}$ metric for the case $\gamma=$ 0 [37]. Note that the two geometric descriptions are not analogous: for $\gamma=0$ (on the diffeomorphism group) the Riemannian exponential map is a local chart, but this is not the case for $\gamma \neq 0$ (on the Bott-Virasoro group) cf. the discussion in the paper [16]. Second, it describes families of pseudo-spherical surfaces [26, 52]. Furthermore, it arises from a non-stretching invariant planar curve flow in the centro-equiaffine geometry [8]. While it is not clear whether the modified $\mathrm{CH}$ equation (1.1) also describes geodesic flows on diffeomorphism groups, we can show that it arises from an intrinsic (arc-length preserving) invariant planar curve flow in Euclidean geometry. Indeed, the modified $\mathrm{KdV}$ and $\mathrm{KdV}$ equations arise naturally from non-stretching invariant planar curve flows in Euclidean and centro-equiaffine geometry, respectively [8, 25, 49].

According to the theory of moving frames, [28, 47], any Euclidean-invariant plane curve flow for $C \subseteq \mathbb{R}^{2}$ has the form

$$
\frac{\partial C}{\partial t}=f \mathbf{n}+g \mathbf{t}
$$

where $\mathbf{t}$ and $\mathbf{n}$ are the Euclidean tangent and normal vectors, while the normal and tangent velocities, $f$ and $g$, are arbitrary Euclidean differential invariants, meaning that they depend on the curvature and its derivatives with respect to the arc-length $s$ of the curve $C$. According to equation (4.13) in [47], which is a consequence of the moving frame calculus developed for curve and submanifold flows invariant under general Lie group actions, the curve flow (2.1) is intrinsic or non-stretching, meaning that it preserves arc length, if and only if

$$
g_{s}-\kappa f=0 \text {. }
$$

In condition (2.2), the operator producing multiplication by $-\kappa$ is particular to Euclidean geometry, and other transformation groups lead to alternative criteria for arc length preservation of the flow. Furthermore, according to Example 5.5 in [47] (see also [25]) when the curve evolves intrinsically according to (2.1) with the constraint (2.2), the curvature invariant satisfies

$$
\kappa_{t}=\mathcal{R}[f], \quad \text { where } \quad \mathcal{R}=\partial_{s}^{2}+\kappa^{2}+\kappa_{s} \partial_{s}^{-1} \kappa
$$

is the well known recursion operator, [46], of the modified $\mathrm{KdV}$ equation

$$
\kappa_{t}=\kappa_{s s s}+\frac{3}{2} \kappa^{2} \kappa_{s}
$$

which corresponds to the particular flow (2.1) with $f=\kappa_{s}, g=\frac{1}{2} \kappa^{2}$.

Remark 2.1. The operator $\mathcal{R}$ is a particular instance, for Euclidean-invariant plane curve flows, of the general moving frame calculus for invariant submanifold flows that has been developed in [47]. For example, the evolution of centro-equiaffine curvature under an invariant curve flow in centro-equiaffine geometry is governed by the KdV recursion operator. In a similar fashion, the operator governing the evolution of curvature and torsion under the 
Euclidean-invariant flow of a space curve is the recursion operator for vortex filament flow, which can be mapped to the integrable nonlinear Schrödinger equation under the Hasimoto transformation, [29, 38]. Many, but not all, Klein geometries produce recursion operators and integrable systems for the evolution of differential invariants under group-invariant flow. It remains an intriguing and unresolved mystery as to why integrable systems arise so often in this geometric context.

In particular, if we set

$$
f=-2 u_{s}, \quad \kappa=m \equiv u-u_{s s},
$$

then by (2.2),

$$
g=-\left(u^{2}-u_{s}^{2}\right)+b,
$$

where $b$ is a constant. Therefore, $u(t, s)$ satisfies the equation

$$
m_{t}+\left(\left(u^{2}-u_{s}^{2}\right) m\right)_{s}+(b+2) u_{s s s}-b u_{s}=0 .
$$

Setting $x=s+(b+2) t,(2.5)$ becomes

$$
m_{t}+\left(\left(u^{2}-u_{x}^{2}\right) m\right)_{x}+2 u_{x}=0, \quad m=u-u_{x x},
$$

which is equivalent, up to rescaling, to the modified $\mathrm{CH}$ equation (1.1). The preceding derivation implies that the modified $\mathrm{CH}$ equation can be regarded as a Euclidean-invariant version of the $\mathrm{CH}$ equation, just as the $\mathrm{mKdV}$ equation is a Euclidean-invariant counterpart to the $\mathrm{KdV}$ equation from the viewpoint of curve flows in Klein geometries.

Since it possesses a recursion operator, the modified $\mathrm{CH}$ equation (1.1) is formally completely integrable. Indeed, it can be written in the bi-Hamiltonian form [48, 51]

$$
m_{t}=-\left(\left(u^{2}-u_{x}^{2}\right) m\right)_{x}-\gamma u_{x}=J \frac{\delta H_{0}}{\delta m}=K \frac{\delta H_{1}}{\delta m},
$$

where

$$
J=-\partial_{x} m \partial_{x}^{-1} m \partial_{x}-\frac{1}{2} \gamma \partial_{x} \quad \text { and } \quad K=\partial_{x}^{3}-\partial_{x}
$$

are compatible Hamiltonian operators, while

$$
H_{0}=\int_{\mathbb{R}} m u d x, \quad H_{1}=\frac{1}{4} \int_{\mathbb{R}}\left(u^{4}+2 u^{2} u_{x}^{2}-\frac{1}{3} u_{x}^{4}+2 \gamma u^{2}\right) d x .
$$

are the corresponding Hamiltonian functionals. According to the Theorem of Magri, [46], the associated recursion operator $\mathcal{R}=J \cdot K^{-1}$ produces a hierarchy of commuting Hamiltonian functionals and bi-Hamiltonian flows - although in this case most are non-local.

The modified $\mathrm{CH}$ equation (1.1) also admits the following Lax formulation [51]:

$$
\left(\begin{array}{l}
\psi_{1} \\
\psi_{2}
\end{array}\right)_{x}=U(m, \lambda)\left(\begin{array}{l}
\psi_{1} \\
\psi_{2}
\end{array}\right), \quad\left(\begin{array}{l}
\psi_{1} \\
\psi_{2}
\end{array}\right)_{t}=V(m, u, \lambda)\left(\begin{array}{l}
\psi_{1} \\
\psi_{2}
\end{array}\right),
$$

where

$$
U(m, \lambda)=\frac{1}{2}\left(\begin{array}{cc}
-Q & \lambda m \\
-\lambda m & Q
\end{array}\right), \quad Q=Q(\lambda, \gamma)=\sqrt{1-\frac{1}{2} \lambda^{2} \gamma}
$$

and

$$
\begin{aligned}
& V(m, u, \lambda)= \\
& \left.\qquad \begin{array}{cc}
\lambda^{-2} Q+\frac{1}{2} Q\left(u^{2}-u_{x}^{2}\right) & -\lambda^{-1}\left(u-Q u_{x}\right)-\frac{1}{2} \lambda\left(u^{2}-u_{x}^{2}\right) m \\
\lambda^{-1}\left(u+Q u_{x}\right)+\frac{1}{2} \lambda\left(u^{2}-u_{x}^{2}\right) m & -\lambda^{-2} Q-\frac{1}{2} Q\left(u^{2}-u_{x}^{2}\right)
\end{array}\right) .
\end{aligned}
$$




\section{ANALYTIC PRELIMINARIES}

In this paper, we will study the Cauchy problem for the modified Camassa-Holm equation on the entire line:

$$
\left\{\begin{array}{l}
m_{t}+\left(\left(u^{2}-u_{x}^{2}\right) m\right)_{x}+\gamma u_{x}=0, \quad m=u-u_{x x} \quad t>0, \quad x \in \mathbb{R}, \\
u(0, x)=u_{0}(x), \quad x \in \mathbb{R} \\
u, m \longrightarrow 0 \quad \text { as } \quad|x| \rightarrow \infty .
\end{array}\right.
$$

Throughout, various positive constants will be uniformly denoted by a common letter $C$. The norm of a Banach space $Z$ is denoted by $\|\cdot\|_{Z}$. Let $\mathcal{S}(\mathbb{R})$ be the space of rapidly decaying $C^{\infty}$ functions on $\mathbb{R}$, and $\mathcal{S}^{\prime}(\mathbb{R})$ its dual space containing all tempered distributions. Given $T>0$, let $C_{c}^{\infty}([0, T) \times \mathbb{R})$ denote the space of all smooth functions with compact support on $[0, T) \times \mathbb{R}$, which may be obtained as the restriction to $[0, T) \times \mathbb{R}$ of smooth functions on $\mathbb{R}^{2}$ with compact support contained in $(-T, T) \times \mathbb{R}$.

We will require the notions of strong (or classical) and weak solutions.

Definition 3.1. If $m \in \mathcal{C}\left([0, T] ; H^{s}\right) \cap \mathcal{C}^{1}\left([0, T] ; H^{s-1}\right)$ with $s>\frac{1}{2}$ and some $T>0$ satisfies (3.1), then $m$ is called a strong solution on $[0, T]$. If $m$ is a strong solution on $[0, T]$ for every $T>0$, then it is called a global strong solution.

Substituting the formula for $m$ in terms of $u$ into the partial differential equation (3.1) results in the following fully nonlinear partial differential equation:

$$
u_{t}+\left(u^{2}-\frac{1}{3} u_{x}^{2}\right) u_{x}+\partial_{x}\left(1-\partial_{x}^{2}\right)^{-1}\left(\frac{2}{3} u^{3}+u u_{x}^{2}+\gamma u\right)+\frac{1}{3}\left(1-\partial_{x}^{2}\right)^{-1} u_{x}^{3}=0 .
$$

Recall that

$$
u=\left(1-\partial_{x}^{2}\right)^{-1} m=p * m, \quad \text { where } \quad p(x)=\frac{1}{2} e^{-|x|},
$$

and $*$ denotes the convolution product on $\mathbb{R}$, defined by

$$
(f * g)(x)=\int_{\mathbb{R}} f(y) g(x-y) d y .
$$

This formulation allows us to define a weak solution as follows.

Definition 3.2. Given initial data $u_{0} \in W^{1,3}(\mathbb{R})$, the function $u \in L_{l o c}^{\infty}\left([0, T), W_{\text {loc }}^{1,3}(\mathbb{R})\right)$ is said to be a weak solution to the initial-value problem (3.1) if it satisfies the following identity:

$$
\begin{gathered}
\int_{0}^{T} \int_{\mathbb{R}}\left[u \varphi_{t}+\frac{1}{3} u^{3} \varphi_{x}+\frac{1}{3} u_{x}^{3} \varphi+p *\left(\frac{2}{3} u^{3}+u u_{x}^{2}+\gamma u\right) \partial_{x} \varphi-\frac{1}{3}\left(p * u_{x}^{3}\right) \varphi\right] d x d t \\
+\int_{\mathbb{R}} u_{0}(x) \varphi(0, x) d x=0
\end{gathered}
$$

for any smooth test function $\varphi(t, x) \in C_{c}^{\infty}([0, T) \times \mathbb{R})$. If $u$ is a weak solution on $[0, T)$ for every $T>0$, then it is called a global weak solution.

Remark 3.1. Since the Sobolev space $W_{\text {loc }}^{1,3}(\mathbb{R})$ can be embedded in the Hölder space $\mathcal{C}^{\alpha}(\mathbb{R})$ with $0 \leq \alpha \leq \frac{2}{3}$, Definition 3.2 precludes the admissibility of discontinuous shock waves as weak solutions.

Wave breaking relies crucially on strong nonlinear dispersion, which, however, makes the analysis more challenging. The conservation of the $H^{1}$-norm allows us to control the solutions to the $\mathrm{CH}$ equation. For transport equations, a solution blows up in finite time 
when its slope $u_{x}$ is unbounded from below. To apply this idea, let us rewrite the modified $\mathrm{CH}$ equation (1.1) as a transport equation in terms of $m$ along the flow generated by $u^{2}-u_{x}^{2}$

$$
m_{t}+\left(u^{2}-u_{x}^{2}\right) m_{x}=-2 u_{x} m^{2}-\gamma u_{x}
$$

Roughly speaking, the transport equation theory ensures that, if the slope

$$
\left(u^{2}-u_{x}^{2}\right)_{x}=2 u_{x} m
$$

is bounded, the solution will remain regular, and can't blow up in finite time. More precisely, the solution blows up at some finite time $T>0$ only if $\|m(t, \cdot)\|_{L^{\infty}}^{2}$ becomes unbounded on $[0, T)$. From this, together with the Sobolev embedding theorem, one can see that the solution blows up in finite time if and only if the slope (3.6) is unbounded from below. Thus to obtain a global solution, the main problem is that it is impossible to be bound (3.6) in terms of the $H^{1}$-norm of the solution unless a higher, positive conserved quantity involved in $H^{3}$-norm of the solution can be found. To overcome this difficulty, we may regard the evolution equation (3.5) in terms of the quantity (3.6) being transported along the flow generated by $u^{2}-u_{x}^{2}$. Then wave breaking can be established by using the global conservative property of the potential density $m$ along the characteristics; see (5.1) below. We expect this new method can be found more applications to deal with wave breaking of the nonlinear dispersive equations with higher nonlinearities.

\section{BLOW-UP CRITERIA}

In this section, we study the well-posedness and establish criteria for the blow up of solutions to the Cauchy problem for the modified $\mathrm{CH}$ equation (3.1). We first recall some 1-D Moser-type estimates, [27].

Proposition 4.1. For $s \geq 0$, the following estimates hold:

$$
\begin{aligned}
\|f g\|_{H^{s}(\mathbb{R})} & \leq C\left(\|f\|_{H^{s}(\mathbb{R})}\|g\|_{L^{\infty}(\mathbb{R})}+\|f\|_{L^{\infty}(\mathbb{R})}\|g\|_{H^{s}(\mathbb{R})}\right), \\
\left\|f \partial_{x} g\right\|_{H^{s}(\mathbb{R})} & \leq C\left(\|f\|_{H^{s+1}(\mathbb{R})}\|g\|_{L^{\infty}(\mathbb{R})}+\|f\|_{L^{\infty}(\mathbb{R})}\left\|\partial_{x} g\right\|_{H^{s}(\mathbb{R})}\right),
\end{aligned}
$$

where the $C$ 's are constants independent of $f$ and $g$.

The following estimates for solutions to the one-dimensional transport equation have been used in [2, 27]. The following result is Theorem 3.14 in [2].

Lemma 4.1. Consider the one-dimensional linear transport equation

$$
\partial_{t} f+v \partial_{x} f=g,\left.\quad f\right|_{t=0}=f_{0} .
$$

Let $0 \leq \sigma<1$, and suppose that

$$
\begin{array}{lll}
f_{0} \in H^{\sigma}(\mathbb{R}), & g \in L^{1}\left([0, T] ; H^{\sigma}(\mathbb{R})\right), \\
v_{x} \in L^{1}\left([0, T] ; L^{\infty}(\mathbb{R})\right), & & f \in L^{\infty}\left([0, T] ; H^{\sigma}(\mathbb{R})\right) \cap \mathcal{C}\left([0, T] ; \mathcal{S}^{\prime}(\mathbb{R})\right) .
\end{array}
$$

Then $f \in \mathcal{C}\left([0, T] ; H^{\sigma}(\mathbb{R})\right)$. More precisely, there exists a constant $C$ depending only on $\sigma$ such that, for every $0<t \leq T$,

$$
\|f(t)\|_{H^{\sigma}} \leq\left\|f_{0}\right\|_{H^{\sigma}}+C \int_{0}^{t}\|g(\tau)\|_{H^{\sigma}} d \tau+C \int_{0}^{t}\|f(\tau)\|_{H^{\sigma}} V^{\prime}(\tau) d \tau
$$

and hence,

$\|f(t)\|_{H^{\sigma}} \leq e^{C V(t)}\left(\left\|f_{0}\right\|_{H^{\sigma}}+C \int_{0}^{t}\|g(\tau)\|_{H^{\sigma}} d \tau\right) \quad$ with $\quad V(t)=\int_{0}^{t}\left\|\partial_{x} v(\tau)\right\|_{L^{\infty}} d \tau$.

In [22], the following local well-posedness result was obtained (with a slight modification). 
Theorem 4.1. Let $m_{0}=\left(1-\partial_{x}^{2}\right) u_{0} \in H^{s}(\mathbb{R})$ with $s>\frac{1}{2}$. Then there exists a time $T>0$ such that the initial-value problem (3.1) has a unique strong solution $m \in \mathcal{C}\left([0, T] ; H^{s}\right) \cap$ $\mathcal{C}^{1}\left([0, T] ; H^{s-1}\right)$ and the map $m_{0} \mapsto m$ is continuous from a neighborhood of $m_{0}$ in $H^{s}$ into $\mathcal{C}\left([0, T] ; H^{s}\right) \cap \mathcal{C}^{1}\left([0, T] ; H^{s-1}\right)$.

We are now in a position to state a blow-up criterion for the modified $\mathrm{CH}$ equation.

Theorem 4.2. Let $m_{0}=\left(1-\partial_{x}^{2}\right) u_{0} \in H^{s}(\mathbb{R})$ be as in Theorem 4.1 with $s>\frac{1}{2}$. Let $m$ be the corresponding solution to (3.1). Assume $T_{m_{0}}^{*}>0$ is the maximum time of existence. Then

$$
T_{m_{0}}^{*}<\infty \Rightarrow \int_{0}^{T_{m_{0}}^{*}}\|m(\tau)\|_{L^{\infty}}^{2} d \tau=\infty .
$$

Remark 4.1. The blow-up criterion (4.4) implies that the lifespan $T_{m_{0}}^{*}$ does not depend on the regularity index $s$ of the initial data $m_{0}$. Indeed, let $m_{0}$ be in $H^{s}$ for some $s>\frac{1}{2}$ and consider some $s^{\prime} \in\left(\frac{1}{2}, s\right)$. Denote by $m_{s}$ (resp., $\left.m_{s^{\prime}}\right)$ the corresponding maximal $H^{s}$ (resp., $H^{s^{\prime}}$ ) solution given by the above theorem. Denote by $T_{s}^{*}\left(\right.$ resp., $\left.T_{s^{\prime}}^{*}\right)$ the lifespan of $m_{s}$ (resp., $\left.m_{s^{\prime}}\right)$. Since $H^{s} \hookrightarrow H^{s^{\prime}}$, uniqueness ensures that $T_{s}^{*} \leq T_{s^{\prime}}^{*}$ and that $m_{s} \equiv m_{s^{\prime}}$ on $\left[0, T_{s}^{*}\right)$. Now, if $T_{s}^{*}<T_{s^{\prime}}^{*}$, then we must have $m_{s^{\prime}}$ in $\mathcal{C}\left(\left[0, T_{s}^{*}\right] ; H^{s^{\prime}}\right)$. Hence, $m_{s^{\prime}} \in$ $L^{2}\left(\left[0, T_{s}^{*}\right] ; L^{\infty}\right)$, which contradicts the above blow-up criterion (4.4). Therefore, $T_{s}^{*}=T_{s^{\prime}}^{*}$.

Proof of Theorem 4.2. We shall prove Theorem 4.2 by an inductive argument with respect to the index $s$. This can be achieved as follows.

Step 1. For $s \in\left(\frac{1}{2}, 1\right)$, applying Lemma 4.1 to equation (3.5), thought of as a transport equation for $m$, we obtain

$$
\begin{aligned}
\|m(t)\|_{H^{s}} \leq & \left\|m_{0}\right\|_{H^{s}}+C \int_{0}^{t}\left\|\left(u^{2}-u_{x}^{2}\right)_{x}(\tau)\right\|_{L^{\infty}}\|m(\tau)\|_{H^{s}} d \tau \\
& +C \int_{0}^{t}\left\|\left(u_{x} m^{2}\right)(\tau)\right\|_{H^{s}} d \tau+C|\gamma| \int_{0}^{t}\left\|u_{x}(\tau)\right\|_{H^{s}} d \tau
\end{aligned}
$$

for all $0<t<T_{m_{0}}^{*}$. Owing to the first Moser-type estimate in (4.1), one has

$$
\begin{aligned}
\left\|u_{x} m^{2}\right\|_{H^{s}} & \leq C\left(\left\|u_{x}\right\|_{H^{s}}\left\|m^{2}\right\|_{L^{\infty}}+\left\|u_{x}\right\|_{L^{\infty}}\left\|m^{2}\right\|_{H^{s}}\right) \\
& \leq C\left(\left\|u_{x}\right\|_{H^{s}}\|m\|_{L^{\infty}}^{2}+\left\|u_{x}\right\|_{L^{\infty}}\|m\|_{L^{\infty}}\|m\|_{H^{s}}\right) .
\end{aligned}
$$

According to (3.3),

$$
u_{x}=\partial_{x}\left(1-\partial_{x}^{2}\right)^{-1} m=\partial_{x} p * m, \quad \text { where } \quad \partial_{x} p(x)=-\frac{1}{2} \operatorname{sign}(x) e^{-|x|} .
$$

Young's inequality implies

$$
\left\|u_{x}\right\|_{L^{\infty}} \leq\left\|\partial_{x} p\right\|_{L^{1}}\|m\|_{L^{\infty}} \leq\|m\|_{L^{\infty}}
$$

which, together with the fact $\left\|u_{x}\right\|_{H^{s}} \leq C\|m\|_{H^{s}}$ and (4.6), gives rise to

$$
\left\|u_{x} m^{2}\right\|_{H^{s}} \leq C\|m\|_{H^{s}}\|m\|_{L^{\infty}}^{2}
$$

and

$$
\left\|\left(u^{2}-u_{x}^{2}\right)_{x}\right\|_{L^{\infty}}=2\left\|m u_{x}\right\|_{L^{\infty}} \leq C\|m\|_{L^{\infty}}\left\|u_{x}\right\|_{L^{\infty}} \leq C\|m\|_{L^{\infty}}^{2} .
$$

Plugging (4.9) into (4.5) leads to

$$
\|m(t)\|_{H^{s}} \leq\left\|m_{0}\right\|_{H^{s}}+C \int_{0}^{t}\left(\|m(\tau)\|_{L^{\infty}}^{2}+|\gamma|\right)\|m(\tau)\|_{H^{s}} d \tau,
$$


which, by Gronwall's inequality, yields

$$
\|m(t)\|_{H^{s}} \leq\left\|m_{0}\right\|_{H^{s}} e^{C \int_{0}^{t}\left(\|m(\tau)\|_{L}^{2}+|\gamma|\right) d \tau} .
$$

Therefore, if the maximal existence time $T_{m_{0}}^{*}<\infty$ satisfies

$$
\int_{0}^{T_{m_{0}}^{*}}\|m(\tau)\|_{L^{\infty}}^{2} d \tau<\infty
$$

the inequality (4.12) implies that

$$
\limsup _{t \rightarrow T_{m_{0}}^{*}}\|m(t)\|_{H^{s}}<\infty,
$$

which contradicts the assumption on the maximal existence time $T_{\mathbf{u}_{0}}^{\star}<\infty$. This completes the proof of Theorem 4.2 for $s \in\left(\frac{1}{2}, 1\right)$.

Step 2. For $s \in[1,2)$, by differentiating (3.5) once with respect to $x$, we have

$$
\partial_{t}\left(m_{x}\right)+\left(u^{2}-u_{x}^{2}\right) \partial_{x}\left(m_{x}\right)=-3 u_{x}\left(m^{2}\right)_{x}-2 u_{x x} m^{2}-\gamma u_{x x} .
$$

Applying Lemma 4.1 to (4.14) yields

$$
\begin{array}{r}
\left\|\partial_{x} m(t, \cdot)\right\|_{H^{s-1}} \leq\left\|\partial_{x} m_{0}\right\|_{H^{s-1}}+C \int_{0}^{t}\left\|\left(u^{2}-u_{x}^{2}\right)_{x}\right\|_{L^{\infty}}\left\|\partial_{x} m\right\|_{H^{s-1}} d \tau \\
+C \int_{0}^{t}\left(\left\|u_{x}\left(m^{2}\right)_{x}\right\|_{H^{s-1}}+\left\|u_{x x} m^{2}\right\|_{H^{s-1}}+|\gamma|\left\|u_{x x}\right\|_{H^{s-1}}\right) d \tau
\end{array}
$$

Thanks to the Moser-type estimates (4.1) along with (4.8), one deduces

$$
\begin{aligned}
\left\|u_{x} \partial_{x}\left(m^{2}\right)\right\|_{H^{s-1}} & \leq C\left(\left\|u_{x}\right\|_{H^{s}}\left\|m^{2}\right\|_{L^{\infty}}+\left\|u_{x}\right\|_{L^{\infty}}\left\|\partial_{x}\left(m^{2}\right)\right\|_{H^{s-1}}\right) \\
& \leq C\left(\left\|m_{x}\right\|_{H^{s-1}}\|m\|_{L^{\infty}}^{2}+\left\|u_{x}\right\|_{L^{\infty}}\|m\|_{L^{\infty}}\|m\|_{H^{s}}\right) \\
& \leq C\|m\|_{L^{\infty}}^{2}\|m\|_{H^{s}} .
\end{aligned}
$$

Using the Moser-type estimates (4.1) again leads to

$$
\begin{aligned}
\| u_{x x} m^{2} & \left\|_{H^{s-1}}+|\gamma|\right\| u_{x x} \|_{H^{s-1}} \\
& \leq C\left(\left\|u_{x x}\right\|_{L^{\infty}}\left\|m^{2}\right\|_{H^{s-1}}+\left\|u_{x x}\right\|_{H^{s-1}}\left\|m^{2}\right\|_{L^{\infty}}+|\gamma|\left\|u_{x x}\right\|_{H^{s-1}}\right) \\
& \leq C\left(\|m\|_{L^{\infty}}^{2}+|\gamma|\right)\|m\|_{H^{s-1}},
\end{aligned}
$$

where we used the fact that

$$
\left\|u_{x x}\right\|_{L^{\infty}} \leq C\left(\|m\|_{L^{\infty}}+\|p * m\|_{L^{\infty}}\right) \leq C\|m\|_{L^{\infty}}
$$

in the last inequality. Using (4.10), (4.16), and (4.17) in (4.15), and combining with (4.11), we conclude that, for $1 \leq s<2$, (4.11) holds.

Repeating the same argument as in Step 1, we see that Theorem 4.2 holds for $1 \leq s<2$.

Step 3. Suppose $2 \leq k \in \mathbb{N}$. By induction, we assume that (4.4) holds when $k-1 \leq s<k$, and prove that it holds for $k \leq s<k+1$. To this end, we differentiate (3.5) $k$ times with respect to $x$, producing

$\partial_{t} \partial_{x}^{k} m+\left(u^{2}-u_{x}^{2}\right) \partial_{x}\left(\partial_{x}^{k} m\right)=-\sum_{\ell=0}^{k-1} C_{k}^{\ell} \partial_{x}^{k-\ell}\left(u^{2}-u_{x}^{2}\right) \partial_{x}^{\ell+1} m-2 \partial_{x}^{k}\left(u_{x} m^{2}\right)-\gamma \partial_{x}^{k} \partial_{x} u$. 
Lemma 4.1 applied to the above again implies that

$$
\begin{aligned}
& \left\|\partial_{x}^{k} m(t)\right\|_{H^{s-k}} \leq\left\|\partial_{x}^{k} m_{0}\right\|_{H^{s-k}}+C \int_{0}^{t}\left\|\partial_{x}^{k} m(\tau)\right\|_{H^{s-k}}\left\|m \partial_{x} u(\tau)\right\|_{L^{\infty}} d \tau \\
& +C \int_{0}^{t}\left\|\left(\sum_{\ell=0}^{k-1} C_{k}^{\ell} \partial_{x}^{k-\ell}\left(u^{2}-u_{x}^{2}\right) \partial_{x}^{\ell} \partial_{x} m+2 \partial_{x}^{k}\left(u_{x} m^{2}\right)+\gamma \partial_{x}^{k} \partial_{x} u\right)(\tau)\right\|_{H^{s-k}} d \tau .
\end{aligned}
$$

Using the first Moser-type estimate in (4.1) and the Sobolev embedding inequality, we have

$$
\begin{aligned}
\| 2 \partial_{x}^{k}\left(u_{x} m^{2}\right) & +\gamma \partial_{x}^{k} \partial_{x} u \|_{H^{s-k}} \\
\leq & C\left(\left\|u_{x}\right\|_{L^{\infty}}\left\|m^{2}\right\|_{H^{s}}+\left\|u_{x}\right\|_{H^{s}}\left\|m^{2}\right\|_{L^{\infty}}+|\gamma|\left\|\partial_{x} u\right\|_{H^{s}}\right) \\
\leq & C\left(\left\|u_{x}\right\|_{L^{\infty}}\|m\|_{L^{\infty}}\|m\|_{H^{s}}+\left\|u_{x}\right\|_{H^{s}}\|m\|_{L^{\infty}}^{2}+|\gamma|\left\|\partial_{x} u\right\|_{H^{s}}\right) \\
\leq & C\left(\|m\|_{L^{\infty}}^{2}+|\gamma|\right)\|m\|_{H^{s}}
\end{aligned}
$$

and

$$
\begin{aligned}
& \left\|\sum_{\ell=0}^{k-1} C_{k}^{\ell} \partial_{x}^{k-\ell}\left(u^{2}-u_{x}^{2}\right) \partial_{x}^{\ell+1} m\right\|_{H^{s-k}} \\
& \leq C \sum_{\ell=0}^{k-1} C_{k}^{\ell}\left(\left\|u^{2}-u_{x}^{2}\right\|_{H^{s-\ell+1}}\left\|\partial_{x}^{\ell} m\right\|_{L^{\infty}}+\left\|\partial_{x}^{k-\ell}\left(u^{2}-u_{x}^{2}\right)\right\|_{L^{\infty}}\|m\|_{H^{s-k+\ell+1}}\right) \\
& \leq C \sum_{\ell=0}^{k-1} C_{k}^{\ell}\left[\left(\|u\|_{L^{\infty}}+\left\|u_{x}\right\|_{L^{\infty}}\right)\left(\|u\|_{H^{s-\ell+1}}+\left\|u_{x}\right\|_{H^{s-\ell+1}}\right)\|m\|_{H^{\ell+\frac{1}{2}+\varepsilon_{0}}}\right. \\
& \left.\quad+\left(\|u\|_{H^{k-\ell+\frac{1}{2}+\varepsilon_{0}}}^{2}+\left\|u_{x}\right\|_{H^{k-\ell+\frac{1}{2}+\varepsilon_{0}}}^{2}\right)\|m\|_{H^{s-k+\ell+1}}\right] \\
& \leq C_{k}\|m\|_{H^{k-\frac{1}{2}+\varepsilon_{0}}}^{2}\|m\|_{H^{s}},
\end{aligned}
$$

where the genius constant $\varepsilon_{0} \in\left(0, \frac{1}{4}\right)$ so that $H^{\frac{1}{2}+\varepsilon_{0}}(\mathbb{R}) \hookrightarrow L^{\infty}(\mathbb{R})$ holds. Substituting (4.20) and (4.21) into (4.19), we derive from (4.11) (with $\frac{1}{2}<s<1$ ) in Step 1 that

$$
\|m(t)\|_{H^{s}} \leq\left\|m_{0}\right\|_{H^{s}}+C \int_{0}^{t}\left(\|m(\tau)\|_{H^{k-\frac{1}{2}+\varepsilon_{0}}}^{2}+|\gamma|\right)\|m(\tau)\|_{H^{s}} d \tau,
$$

where we used the Sobolev embedding theorem $H^{k-\frac{1}{2}+\varepsilon_{0}}(\mathbb{R}) \hookrightarrow L^{\infty}(\mathbb{R})$ with $k \geq 2$.

Applying Gronwall's inequality then gives

$$
\|m(t)\|_{H^{s}} \leq\left\|m_{0}\right\|_{H^{s}} \exp \left\{C \int_{0}^{t}\left(\|m(\tau)\|_{H^{k-\frac{1}{2}+\varepsilon_{0}}}^{2}+|\gamma|\right) d \tau\right\} .
$$

In consequence, if the maximal existence time $T_{\mathbf{u}_{0}}^{\star}<\infty$ satisfies

$$
\int_{0}^{T_{\mathbf{u}_{0}}^{\star}}\|m(\tau)\|_{L^{\infty}}^{2} d \tau<\infty,
$$

thanks to the uniqueness of solution in Theorem 4.1, we then find that $\|m(t)\|_{H^{k-\frac{1}{2}+\varepsilon_{0}}}$ is uniformly bounded in $t \in\left(0, T_{\mathbf{m}_{0}}^{*}\right)$ by the induction assumption, which along with (4.23) implies

$$
\limsup _{t \rightarrow T_{\mathbf{m}_{0}}^{*}}\|m(t)\|_{H^{s}}<\infty
$$

which leads to a contradiction. 
Therefore, Steps 1 to 3 complete the proof of Theorem 4.2.

Remark 4.2. For a strong solution $m=u-u_{x x}$ in Theorem 4.1, the Hamiltonian functionals (2.7) are conserved, that is

$$
\frac{d}{d t} \int_{\mathbb{R}}\left(u^{2}+u_{x}^{2}\right) d x=0, \quad \frac{d}{d t} \int_{\mathbb{R}}\left(u^{4}+2 u^{2} u_{x}^{2}-\frac{1}{3} u_{x}^{4}+2 \gamma u^{2}\right) d x=0,
$$

for all $t \in[0, T)$.

The following blow-up criterion demonstrates that wave-breaking depends only on the infimum of $m u_{x}$.

Theorem 4.3. Let $m_{0} \in H^{s}(\mathbb{R})$ be as in Theorem 4.1 with $s>\frac{1}{2}$. Then the corresponding solution $m$ to (3.1) blows up in finite time $T_{m_{0}}^{*}>0$ if and only if

$$
\lim _{t \rightarrow T_{m_{0}}^{*}} \inf _{x \in \mathbb{R}}\left\{\left(m u_{x}\right)(t, x)\right\}=-\infty
$$

Proof. Since, in view of Remark 4.1, the existence time $T_{m_{0}}^{*}$ is independent of the choice of $s$, we need only to consider the case $s=3$, which relies on a simple density argument.

Multiplying equation (3.5) by $m$ and integrating over $\mathbb{R}$ with respect to $x$, and then integration by parts, produces

$$
\begin{aligned}
\frac{1}{2} \frac{d}{d t} \int_{\mathbb{R}} m^{2} d x & =-\int_{\mathbb{R}}\left(u^{2}-u_{x}^{2}\right) m m_{x} d x-2 \int_{\mathbb{R}} u_{x} m^{3} d x-\gamma \int_{\mathbb{R}} u_{x} m d x \\
& =\frac{1}{2} \int_{\mathbb{R}}\left(u^{2}-u_{x}^{2}\right)_{x} m^{2} d x-2 \int_{\mathbb{R}} u_{x} m^{3} d x-\frac{\gamma}{2} \int_{\mathbb{R}}\left(u^{2}-u_{x}^{2}\right)_{x} d x \\
& =-\int_{\mathbb{R}}\left(m u_{x}\right) m^{2} d x
\end{aligned}
$$

We next expand out (4.14):

$$
\begin{aligned}
m_{x t} & =-2 u_{x x} m^{2}-6 u_{x} m m_{x}-\left(u^{2}-u_{x}^{2}\right) m_{x x}-\gamma u_{x x} \\
& =-2 u m^{2}+2 m^{3}-6 u_{x} m m_{x}-\left(u^{2}-u_{x}^{2}\right) m_{x x}-\gamma u_{x x} .
\end{aligned}
$$

Multiplying by $m_{x}$ and integrating over $\mathbb{R}$ with respect to $x$, leads to

$$
\begin{aligned}
\frac{1}{2} \frac{d}{d t} \int_{\mathbb{R}} m_{x}^{2} d x=-\int_{\mathbb{R}}\left(u^{2}-u_{x}^{2}\right) m_{x} m_{x x} d x-2 \int_{\mathbb{R}} u m^{2} m_{x} d x \\
\quad-6 \int_{\mathbb{R}} u_{x} m m_{x}^{2} d x+2 \int_{\mathbb{R}} m^{3} m_{x} d x-\gamma \int_{\mathbb{R}} u_{x x} m_{x} d x \\
=\frac{1}{2} \int_{\mathbb{R}}\left(u^{2}-u_{x}^{2}\right)_{x} m_{x}^{2} d x+\frac{2}{3} \int_{\mathbb{R}} u_{x} m^{3} d x \\
\quad-6 \int_{\mathbb{R}} u_{x} m m_{x}^{2} d x-\frac{\gamma}{2} \int_{\mathbb{R}}\left(u_{x}^{2}-u_{x x}^{2}\right)_{x} d x \\
=-5 \int_{\mathbb{R}} u_{x} m m_{x}^{2} d x+\frac{2}{3} \int_{\mathbb{R}} u_{x} m^{3} d x .
\end{aligned}
$$

Therefore,

$$
\frac{d}{d t} \int_{\mathbb{R}}\left(m^{2}+m_{x}^{2}\right) d x=-10 \int_{\mathbb{R}}\left(m u_{x}\right) m_{x}^{2} d x-\frac{2}{3} \int_{\mathbb{R}}\left(m u_{x}\right) m^{2} d x .
$$

If $m u_{x}$ is bounded from below on $\left[0, T_{m_{0}}^{*}\right) \times \mathbb{R}$, i.e., there exists a positive constant $C_{1}>0$ such that $m u_{x} \geq-C_{1}$ on $\left[0, T_{m_{0}}^{*}\right) \times \mathbb{R}$, then the above estimate implies

$$
\frac{d}{d t} \int_{\mathbb{R}}\left(m^{2}+m_{x}^{2}\right) d x \leq 10 C_{1} \int_{\mathbb{R}}\left(m^{2}+m_{x}^{2}\right) d x
$$


Applying Gronwall's inequality then yields

$$
\|m(t)\|_{H^{1}}^{2} \leq \int_{\mathbb{R}}\left(m^{2}+m_{x}^{2}\right) d x \leq e^{10 C_{1} t}\left\|m_{0}\right\|_{H^{1}}^{2}
$$

for $t \in\left[0, T_{m_{0}}^{*}\right)$, which ensures that the solution $m(t, x)$ does not blow up in finite time.

On the other hand, if

$$
\liminf _{t \uparrow T}\left[\inf _{x \in \mathbb{R}}\left(m u_{x}(t, x)\right)\right]=-\infty
$$

by Theorem 4.1 for the existence of local strong solutions and the Sobolev embedding theorem, we infer that the solution will blow-up in finite time. The proof of Theorem 4.3 is then complete.

\section{WAVE-BREAKING MECHANISM IN THE CASE $\gamma=0$}

In this section, we derive some sufficient conditions for the breaking of waves for the initial-value problem (3.1) with the parameter $\gamma=0$, an assumption that, we emphasize, will hold for the remainder of the paper.

For this purpose, a conservative property of the potential $m$ will be crucial in the proofs of our blow-up results. Consider the ordinary differential equation

$$
\left\{\begin{aligned}
\frac{d q(t, x)}{d t} & =\left(u^{2}-u_{x}^{2}\right)(t, q(t, x)), \quad x \in \mathbb{R}, \quad t \in[0, T), \\
q(0, x) & =x
\end{aligned}\right.
$$

for the flow generated by $u^{2}-u_{x}^{2}$.

Lemma 5.1. Let $u_{0} \in H^{s}(\mathbb{R}), s>\frac{5}{2}$, and let $T>0$ be the maximal existence time of the corresponding strong solution $u$ to (3.1). Then (5.1) has a unique solution $q \in$ $\mathcal{C}^{1}([0, T) \times \mathbb{R}, \mathbb{R})$ such that the map $q(t, \cdot)$ is an increasing diffeomorphism of $\mathbb{R}$ with

$$
q_{x}(t, x)=\exp \left(2 \int_{0}^{t}\left(m u_{x}\right)(s, q(s, x)) d s\right)>0, \quad \text { for all } \quad(t, x) \in[0, T) \times \mathbb{R} .
$$

Furthermore,

$$
m(t, q(t, x)) q_{x}(t, x)=m_{0}(x), \quad \text { for all } \quad(t, x) \in[0, T) \times \mathbb{R} .
$$

Proof. Since $u \in \mathcal{C}^{1}\left([0, T), H^{s-1}(\mathbb{R})\right)$ and $H^{s}(\mathbb{R}) \hookrightarrow \mathcal{C}^{1}(\mathbb{R})$, both $u(t, x)$ and $u_{x}(t, x)$ are bounded, Lipschitz in the space variable $x$, and of class $\mathcal{C}^{1}$ in time. Therefore, by well-known classical results in the theory of ordinary differential equations, the initial value problem (5.1) has a unique solution $q(t, x) \in \mathcal{C}^{1}([0, T) \times \mathbb{R})$.

Differentiating (5.1) with respect to $x$ and using (3.6) yields

$$
\left\{\begin{array}{l}
\frac{d}{d t} q_{x}=2\left(m u_{x}\right)(t, q) q_{x}, \\
q_{x}(0, x)=1,
\end{array} \quad(t, x) \in[0, T) \times \mathbb{R} .\right.
$$

The solution to (5.4) is given by

$$
q_{x}(t, x)=\exp \left(2 \int_{0}^{t}\left(m u_{x}\right)(s, q(s, x)) d s\right), \quad(t, x) \in[0, T) \times \mathbb{R} .
$$

For every $T^{\prime}<T$, it follows from the Sobolev embedding theorem that

$$
\sup _{(s, x) \in\left[0, T^{\prime}\right) \times \mathbb{R}}\left|\left(m u_{x}\right)(s, x)\right|<\infty
$$


We infer from (5.5) that there exists a constant $K>0$ such that $q_{x}(t, x) \geq e^{-K t},(t, x) \in$ $[0, T) \times \mathbb{R}$, which implies that the map $q(t, \cdot)$ is an increasing diffeomorphism of $\mathbb{R}$ before blow-up with

$$
q_{x}(t, x)=\exp \left(2 \int_{0}^{t}\left(m u_{x}\right)(s, q(s, x)) d s\right)>0, \quad \text { for all } \quad(t, x) \in[0, T) \times \mathbb{R} .
$$

On the other hand, combining (5.4) with (1.1), we have

$$
\begin{aligned}
\frac{\partial}{\partial t}\left[m(t, q(t, x)) q_{x}(t, x)\right] & =\left[m_{t}(t, q)+m_{x}(t, q) q_{t}\right] q_{x}+m q_{x t} \\
& =q_{x}\left[m_{t}(t, q)+\left(u^{2}-u_{x}^{2}\right) m_{x}(t, q)\right]+2 u_{x} m^{2} q_{x} \\
& =q_{x}\left[m_{t}+\left(u^{2}-u_{x}^{2}\right) m_{x}+2 u_{x} m^{2}\right]=0 .
\end{aligned}
$$

Therefore, $m(t, q(t, x)) q_{x}(t, x)$ is independent of the time variable $t$. That is

$$
m(t, q(t, x)) q_{x}(t, x)=m(0, x)=u_{0}(x)-u_{0 x x}(x) .
$$

This completes the proof of Lemma 5.1.

Remark 5.1. Lemma 5.1 shows that, if $m_{0}=\left(1-\partial_{x}^{2}\right) u_{0}$ does not change sign, then $m(t, x)$ will not change sign for any $t \in[0, T)$.

Remark 5.2. Since $q(t, \cdot): \mathbb{R} \rightarrow \mathbb{R}$ is a diffeomorphism of the line for every $t \in[0, T)$, the $L^{\infty}$-norm of any function $v(t, \cdot) \in L^{\infty}$ is preserved under the family of diffeomorphisms $q(t, \cdot)$, that is,

$$
\|v(t, \cdot)\|_{L^{\infty}}=\|v(t, q(t, \cdot))\|_{L^{\infty}}, \quad t \in[0, T) .
$$

Proposition 5.1. Let $m_{0} \in H^{s}(\mathbb{R}), s \geq 3$ and $T>0$ be the maximal time of existence of the corresponding solution $m(t, x)$ to (3.1) with the initial data $m_{0}$. Then $M:=u_{x} m$ satisfies

$$
M_{t}+\left(u^{2}-u_{x}^{2}\right) M_{x}=-2 M^{2}-2 m\left(1-\partial_{x}^{2}\right)^{-1}\left(u_{x}^{2} m\right)-2 m \partial_{x}\left(1-\partial_{x}^{2}\right)^{-1}\left(u u_{x} m\right)
$$

for all $(t, x) \in[0, T) \times \mathbb{R}$.

Proof. From (1.1), we have

$$
\begin{aligned}
\left(1-\partial_{x}^{2}\right) & {\left[u_{t}+\left(u^{2}-u_{x}^{2}\right) u_{x}\right] } \\
= & -\left(u^{2}-u_{x}^{2}\right) m_{x}-2 u_{x} m^{2}+\left(1-\partial_{x}^{2}\right)\left[\left(u^{2}-u_{x}^{2}\right) u_{x}\right] \\
= & -\left(u^{2}-u_{x}^{2}\right) m_{x}-2 u_{x} m^{2}+\left(u^{2}-u_{x}^{2}\right) u_{x}-\partial_{x}^{2}\left[\left(u^{2}-u_{x}^{2}\right) u_{x}\right] \\
= & -\left(u^{2}-u_{x}^{2}\right) m_{x}-2 u_{x} m^{2}+\left(u^{2}-u_{x}^{2}\right) m_{x}-6 u_{x} u_{x x} m-2 u_{x}^{2} m_{x} \\
= & -2 u_{x} m^{2}-6 u_{x} u_{x x} m-2 u_{x}^{2} m_{x},
\end{aligned}
$$

which implies

$$
\begin{aligned}
u_{t}+\left(u^{2}-u_{x}^{2}\right) u_{x} & =-\left(1-\partial_{x}^{2}\right)^{-1}\left[2 u_{x} m^{2}+6 u_{x} u_{x x} m+2 u_{x}^{2} m_{x}\right] \\
& =-\left(1-\partial_{x}^{2}\right)^{-1}\left[\left(u^{2}\right)_{x} m+2\left(u_{x}^{2} m\right)_{x}\right] .
\end{aligned}
$$

Taking the derivative to (5.8) with respect to $x$ yields

$$
u_{x t}+2 u_{x}^{2} m+\left(u^{2}-u_{x}^{2}\right) u_{x x}=-\partial_{x}\left(1-\partial_{x}^{2}\right)^{-1}\left[\left(u^{2}\right)_{x} m+2\left(u_{x}^{2} m\right)_{x}\right] .
$$

Notice that $m_{t}=-\left(u^{2}-u_{x}^{2}\right) m_{x}-2 u_{x} m^{2}$. We deduce from (5.9) that

$$
m u_{x t}=-2 u_{x}^{2} m^{2}-\left(u^{2}-u_{x}^{2}\right) u_{x x} m-m \partial_{x}\left(1-\partial_{x}^{2}\right)^{-1}\left[\left(u^{2}\right)_{x} m+2\left(u_{x}^{2} m\right)_{x}\right] .
$$

Clearly,

$$
u_{x} m_{t}=-\left(u^{2}-u_{x}^{2}\right) u_{x} m_{x}-2 u_{x}^{2} m^{2} .
$$


Hence, we obtain the following equation for $M=m u_{x}$ :

$$
\begin{aligned}
M_{t} & +\left(u^{2}-u_{x}^{2}\right) M_{x}=-4 M^{2}-m \partial_{x}\left(1-\partial_{x}^{2}\right)^{-1}\left[\left(u^{2}\right)_{x} m+2\left(u_{x}^{2} m\right)_{x}\right] \\
& =-4 M^{2}-m \partial_{x}\left(1-\partial_{x}^{2}\right)^{-1}\left(\left(u^{2}\right)_{x} m\right)-2 m \partial_{x}^{2}\left(1-\partial_{x}^{2}\right)^{-1}\left(u_{x}^{2} m\right) \\
& =-4 M^{2}-m \partial_{x}\left(1-\partial_{x}^{2}\right)^{-1}\left(\left(u^{2}\right)_{x} m\right)+m\left(2 u_{x}^{2} m\right)-m\left(1-\partial_{x}^{2}\right)^{-1}\left(2 u_{x}^{2} m\right) \\
& =-2 M^{2}-2 m \partial_{x}\left(1-\partial_{x}^{2}\right)^{-1}\left(u u_{x} m\right)-2 m\left(1-\partial_{x}^{2}\right)^{-1}\left(u_{x}^{2} m\right) .
\end{aligned}
$$

This completes the proof of Proposition 5.1.

Lemma 5.2. Let $T>0$ be the maximal time of existence of the solution $m(t, x)$ to the initial value problem (3.1) with initial data $m_{0} \in H^{s}(\mathbb{R})$ for $s \geq 3$. Assume $m_{0} \geq 0$ for all $x \in \mathbb{R}$. Then

$$
\left|u_{x}(t, x)\right| \leq u(t, x), \quad M_{t}+\left(u^{2}-u_{x}^{2}\right) M_{x} \leq-2 M^{2}+2 C_{1} m,
$$

for all $(t, x) \in[0, T) \times \mathbb{R}$, with $C_{1}=\frac{1}{\sqrt{2}}\left\|u_{0}\right\|_{H^{1}}^{3}$.

Proof. Since $m_{0}(x) \geq 0$ for all $x \in \mathbb{R},(5.3)$ and (5.2) imply that

$$
m(t, x) \geq 0,
$$

for all $t \in[0, T), x \in \mathbb{R}$, and hence

$$
\left(m\left(1-\partial_{x}^{2}\right)^{-1}\left(u_{x}^{2} m\right)\right)(t, x) \geq 0 .
$$

According to (3.3),

$$
u(t, x)=(p * m)(t, x)=\frac{1}{2} \int_{\mathbb{R}} e^{-|x-y|} m(t, y) d y,
$$

hence

$$
\begin{aligned}
u(t, x) & =\frac{e^{-x}}{2} \int_{-\infty}^{x} e^{y} m(t, y) d y+\frac{e^{x}}{2} \int_{x}^{+\infty} e^{-y} m(t, y) d y, \\
u_{x}(t, x) & =-\frac{e^{-x}}{2} \int_{-\infty}^{x} e^{y} m(t, y) d y+\frac{e^{x}}{2} \int_{x}^{+\infty} e^{-y} m(t, y) d y,
\end{aligned}
$$

which, along with (5.12), leads to

$$
\begin{aligned}
& u(t, x)+u_{x}(t, x)=e^{x} \int_{x}^{+\infty} e^{-y} m(t, y) d y \geq 0, \\
& u(t, x)-u_{x}(t, x)=e^{-x} \int_{-\infty}^{x} e^{y} m(t, y) d y \geq 0 .
\end{aligned}
$$

From this, we have

$$
\left|u_{x}(t, x)\right| \leq u(t, x) \quad \text { for all } \quad(t, x) \in[0, T) \times \mathbb{R} .
$$

On the other hand, by (4.7),

$$
\begin{aligned}
\partial_{x}\left(1-\partial_{x}^{2}\right)^{-1}\left(u u_{x} m\right)(t, x) & =\partial_{x} p *\left(u u_{x} m\right)(t, x) \\
& =-\frac{1}{2} \int_{-\infty}^{+\infty} \operatorname{sign}(x-y) e^{-|x-y|}\left(u u_{x} m\right)(t, y) d y,
\end{aligned}
$$

which, together with (5.16), implies

$$
\begin{aligned}
\left|\partial_{x}\left(1-\partial_{x}^{2}\right)^{-1}\left(u u_{x} m\right)(t, x)\right| \leq \frac{1}{2} \int_{-\infty}^{+\infty} e^{-|x-y|}\left(\left|u_{x}\right| u m\right)(t, y) d y \\
\leq \frac{1}{2}\|u(t)\|_{L^{\infty}}^{2} \int_{-\infty}^{+\infty} e^{-|x-y|} m(t, y) d y \leq\|u(t)\|_{L^{\infty}}^{3} .
\end{aligned}
$$


From this, together with the Sobolev inequality $\|u\|_{L^{\infty}(\mathbb{R})} \leq \frac{1}{\sqrt{2}}\|u\|_{H^{1}(\mathbb{R})}$ and (4.24), we find

$$
\left|\partial_{x}\left(1-\partial_{x}^{2}\right)^{-1}\left(u u_{x} m\right)(t, x)\right| \leq\left(\frac{1}{\sqrt{2}}\|u(t)\|_{H^{1}}\right)^{3}=\frac{1}{2 \sqrt{2}}\left\|u_{0}\right\|_{H^{1}}^{3}:=C_{1} .
$$

Therefore, plugging (5.13) and (5.18) in (5.6) leads to the second inequality in (5.11), which ends the proof of Lemma 5.2.

In view of Theorem 4.3, we find that the solution blows up in finite time if and only if the slope $u_{x} m$ is unbounded blow. The next theorem shows that if the initial potential $0 \not \equiv m_{0}(x)$ is non-negative, then the slope $u_{x} m$ has an uniform upper bound, independent of the time $t$, as long as $m(t, x)$ exists.

Theorem 5.1. Let $m_{0} \in H^{s}(\mathbb{R}), s>1 / 2$ and $T>0$ be the maximal time of existence of the corresponding solution $m(t, x)$ to (3.1) with the initial data $m_{0}$. Assume that $m_{0}(x)=$ $\left(1-\partial_{x}^{2}\right) u_{0} \geq 0$ for all $x \in \mathbb{R}$, and $m_{0}\left(x_{0}\right)>0$ at some point $x_{0} \in \mathbb{R}$. Then

$$
\sup _{x \in \mathbb{R}}\left(m \partial_{x} u\right)(t, x) \leq \frac{1}{\sqrt{2}}\left\|u_{0}\right\|_{H^{1}} \sup _{x \in \mathbb{R}} m_{0}(x)
$$

for all $t \in[0, T)$.

To prove Theorem 5.1, we need the following lemma due to Constantin and Escher [11].

Lemma 5.3. [11] Let $T>0$ and $v \in \mathcal{C}^{1}\left([0, T) ; H^{2}(\mathbb{R})\right)$. Then for every $t \in[0, T)$, there exists at least one point $\xi(t) \in \mathbb{R}$ with

$$
I(t):=\inf _{x \in \mathbb{R}}\left(v_{x}(t, x)\right)=v_{x}(t, \xi(t)) .
$$

The function $I(t)$ is absolutely continuous on $(0, T)$ with

$$
\frac{d I(t)}{d t}=v_{t x}(t, \xi(t)), \text { a.e.on }(0, T) .
$$

Proof of Theorem 5.1. As in the proof of Theorem 4.3, we only need to show Theorem 5.1 holds when $s=3$. First, Theorem 4.1 implies that $M \in \mathcal{C}\left([0, T) ; H^{s}\right) \cap \mathcal{C}^{1}\left([0, T) ; H^{s-1}\right)$. Given $t \in[0, T)$, let $x_{0}(t) \in \mathbb{R}$ be such that

$$
M\left(t, x_{0}(t)\right)=\sup _{x \in \mathbb{R}} M(t, x), \quad \text { which implies } \quad M_{x}\left(t, x_{0}(t)\right)=0 \text {, a.e. on }(0, T) .
$$

Since $s>\frac{1}{2}$, we have $H^{s}(\mathbb{R}) \hookrightarrow \mathcal{C}_{0}(\mathbb{R})$, the space of all continuous functions on $\mathbb{R}$ vanishing as $|x| \rightarrow \infty$. Theorem 4.1 implies that

$$
M\left(t, x_{0}(t)\right) \geq 0 \quad \text { for all } \quad t \in[0, T) .
$$

Thanks to Lemma 5.1, the map $q(t, \cdot)$ is an increasing diffeomorphism of $\mathbb{R}$, which implies that there is $y_{0}=y_{0}(t) \in \mathbb{R}$ satisfying

$$
q\left(t, y_{0}(t)\right)=x_{0}(t) .
$$

Hence, in view of Lemma 5.3, it follows from (5.6) and (5.20) that

$$
\begin{aligned}
& \frac{d}{d t} M\left(t, x_{0}(t)\right)=- 2 M^{2}\left(t, x_{0}(t)\right)-2\left[m\left(1-\partial_{x}^{2}\right)^{-1}\left(u_{x}^{2} m\right)\right]\left(t, x_{0}(t)\right) \\
&-2\left[m \partial_{x}\left(1-\partial_{x}^{2}\right)^{-1}\left(u u_{x} m\right)\right]\left(t, x_{0}(t)\right), \text { a.e. on }(0, T)
\end{aligned}
$$

which, together with (5.13) and (5.18), leads to

$$
\frac{d}{d t} M\left(t, x_{0}(t)\right) \leq-2 M^{2}\left(t, x_{0}(t)\right)+2 C_{1} m\left(t, x_{0}(t)\right), \quad \text { a.e. } \quad \text { on } \quad(0, T) .
$$


On the other hand, at the point $\left(t, q\left(t, y_{0}\right)\right)=\left(t, x_{0}(t)\right)$, we have

$$
\frac{d}{d t} m\left(t, x_{0}(t)\right)=-2(m M)\left(t, x_{0}(t)\right), \quad \text { a.e. } \quad \text { on } \quad(0, T) .
$$

It then follows from (5.21) that for all $t \in[0, T)$

$$
m\left(t, x_{0}(t)\right)=m_{0}\left(x_{0}(0)\right) e^{-2 \int_{0}^{t} M\left(\tau, x_{0}(\tau)\right) d \tau} \leq m_{0}\left(x_{0}(0)\right) \leq \sup _{x \in \mathbb{R}} m_{0}(x),
$$

which along with (5.11), (4.24) and (5.26) implies

$$
\begin{aligned}
M\left(t, x_{0}(t)\right) & =u_{x}\left(t, x_{0}(t)\right) m\left(t, x_{0}(t)\right) \leq\left\|u_{x}(t)\right\|_{L^{\infty}} m\left(t, x_{0}(t)\right) \\
& \leq\|u(t)\|_{L^{\infty}} m\left(t, x_{0}(t)\right) \leq \frac{1}{\sqrt{2}}\left\|u_{0}\right\|_{H^{1}} \sup _{x \in \mathbb{R}} m_{0}(x) .
\end{aligned}
$$

This completes the proof of Theorem 5.1.

We are now in a position to state the following wave-breaking result.

Theorem 5.2. Suppose $m_{0} \in H^{s}(\mathbb{R})$ with $s>1 / 2$. Let $T>0$ be the maximal time of existence of the corresponding solution $m(t, x)$ to (3.1) with the initial data $m_{0}$. Assume $m_{0}(x)=\left(1-\partial_{x}^{2}\right) u_{0} \geq 0$ for all $x \in \mathbb{R}$ and $m_{0}\left(x_{0}\right)>0$ for some $x_{0} \in \mathbb{R}$, and that

$$
\partial_{x} u_{0}\left(x_{0}\right)<-\left(\frac{\sqrt{2}\left\|u_{0}\right\|_{H^{1}}^{3}}{m_{0}\left(x_{0}\right)}\right)^{\frac{1}{2}}
$$

Then the solution $m(t, x)$ blows up at a time

$$
T_{0} \leq t^{*}:=\frac{-\partial_{x} u_{0}\left(x_{0}\right)}{\sqrt{2}\left\|u_{0}\right\|_{H^{1}}^{3}}-\frac{1}{2} \sqrt{\left(\frac{\sqrt{2} \partial_{x} u_{0}\left(x_{0}\right)}{\left\|u_{0}\right\|_{H^{1}}^{3}}\right)^{2}-\frac{2 \sqrt{2}}{\left\|u_{0}\right\|_{H^{1}}^{3} m_{0}\left(x_{0}\right)}} .
$$

Moreover when $T_{0}=t^{*}$, the following estimate of the blow-up rate holds

$$
\liminf _{t \rightarrow T_{0}^{-}}\left(\left(T_{0}-t\right) \inf _{x \in \mathbb{R}} M(t, x)\right) \leq-1
$$

Proof. Thanks to (5.11) and (5.1), we have

$$
\frac{d}{d t} M\left(t, q\left(t, x_{0}\right)\right) \leq-2 M^{2}\left(t, q\left(t, x_{0}\right)\right)+2 C_{1} m\left(t, q\left(t, x_{0}\right)\right) .
$$

Similarly, one can see from the equation in (3.1) that

$$
\frac{d}{d t} m\left(t, q\left(t, x_{0}\right)\right)=-2 m M\left(t, q\left(t, x_{0}\right)\right)
$$

Denoting $\bar{M}(t):=2 M\left(t, q\left(t, x_{0}\right)\right)$ and $\bar{m}(t):=2 m\left(t, q\left(t, x_{0}\right)\right)$, we reformulate (5.31) and (5.32) as

$$
\frac{d}{d t} \bar{M}(t) \leq-\bar{M}(t)^{2}+2 C_{1} \bar{m}(t)
$$

and

$$
\frac{d}{d t} \bar{m}(t)=-\bar{m}(t) \bar{M}(t) .
$$


Combining this with (5.12), we deduce that

$$
\begin{aligned}
\frac{d}{d t}\left(\frac{1}{\bar{m}(t)^{2}}\right. & \left.\frac{d}{d t} \bar{m}(t)\right)=\frac{d}{d t}\left(-\frac{1}{\bar{m}(t)} \bar{M}(t)\right) \\
& =\frac{1}{\bar{m}(t)^{2}}\left(-\bar{m}(t) \frac{d}{d t} \bar{M}(t)+\bar{M}(t) \frac{d}{d t} \bar{m}(t)\right) \\
& \geq \frac{1}{\bar{m}(t)^{2}}\left(\bar{m}(t)\left(\bar{M}(t)^{2}-2 C_{1} \bar{m}(t)\right)-\bar{m}(t) \bar{M}(t)^{2}\right)=-2 C_{1} .
\end{aligned}
$$

Integrating from 0 to $t$ leads to

$$
\frac{1}{\bar{m}(t)^{2}} \frac{d}{d t} \bar{m}(t) \geq C_{0}-2 C_{1} t
$$

with

$$
C_{0}:=\frac{\bar{m}(0)^{\prime}}{\bar{m}(0)^{2}}=-\frac{\bar{M}(0)}{\bar{m}(0)}=-\left(\partial_{x} u_{0}\right)\left(x_{0}\right)
$$

Combining this with (5.34) yields

$$
\bar{M}(t)=-\frac{1}{\bar{m}(t)} \frac{d}{d t} \bar{m}(t) \leq-\bar{m}(t)\left(C_{0}-2 C_{1} t\right) .
$$

Integrating (5.36) again on $[0, t]$ implies

$$
\frac{1}{\bar{m}(t)}-\frac{1}{\bar{m}(0)} \leq C_{1} t^{2}-C_{0} t
$$

and hence

$$
\frac{1}{\bar{m}(t)} \leq C_{1}\left(t^{2}-\frac{C_{0}}{C_{1}} t+\frac{1}{C_{1} \bar{m}(0)}\right)=C_{1}\left(t^{2}-\frac{C_{0}}{C_{1}} t+\frac{1}{2 C_{1} m_{0}\left(x_{0}\right)}\right) .
$$

The quadratic equation

has two roots:

$$
t^{2}-\frac{C_{0}}{C_{1}} t+\frac{1}{2 C_{1} m_{0}\left(x_{0}\right)}=0
$$

$$
t^{*}:=\frac{C_{0}}{2 C_{1}}-\frac{1}{2} \sqrt{\left(\frac{C_{0}}{C_{1}}\right)^{2}-\frac{2}{C_{1} m_{0}\left(x_{0}\right)}}, \quad t_{*}:=\frac{C_{0}}{2 C_{1}}+\frac{1}{2} \sqrt{\left(\frac{C_{0}}{C_{1}}\right)^{2}-\frac{2}{C_{1} m_{0}\left(x_{0}\right)}},
$$

Assumption (5.28) implies

$$
\left(\frac{C_{0}}{C_{1}}\right)^{2}>\frac{2}{C_{1} m_{0}\left(x_{0}\right)}, \quad \text { hence } \quad 0<t^{*}<\frac{C_{0}}{2 C_{1}}<t_{*} .
$$

Thus,

$$
0 \leq \frac{1}{\bar{m}(t)} \leq C_{1}\left(t-t^{*}\right)\left(t-t_{*}\right)
$$

From this, we may find a time $0<T_{0} \leq t^{*}$ such that

$$
m(t) \longrightarrow+\infty, \quad \text { as } t \longrightarrow T_{0} \leq t^{*},
$$

which, by (5.37), implies that

$$
M(t) \longrightarrow-\infty, \quad \text { as } \quad t \longrightarrow T_{0} \leq t^{*} .
$$

Therefore,

$$
\inf _{x \in \mathbb{R}} M(t, x) \leq M(t) \longrightarrow-\infty, \quad \text { as } \quad t \longrightarrow T_{0} \leq t^{*},
$$

which, in view of Theorem 4.3, implies that the solution $m(t, x)$ blows up at the time $T_{0}$. 
Having established wave breaking results for (3.1) as above, attention is given to blow-up rate for the solution. In fact, owing to (5.37) and (5.41), we derive that for all $0<t<T_{0}$

$$
\begin{aligned}
\left(T_{0}-t\right) \inf _{x \in \mathbb{R}} M(t, x) & \leq\left(T_{0}-t\right) M(t) \leq-\left(T_{0}-t\right) \bar{m}(t)\left(C_{0}-2 C_{1} t\right) \\
& \leq\left(T_{0}-t\right) \frac{1}{C_{1}\left(t-t^{*}\right)\left(t-t_{*}\right)}\left(2 C_{1} t-C_{0}\right) \\
& \leq \frac{2\left(T_{0}-t\right)}{\left(t-t^{*}\right)\left(t-t_{*}\right)}\left(t-\frac{C_{0}}{2 C_{1}}\right),
\end{aligned}
$$

which leads to (5.30) when $T_{0}=t^{*}$. Therefore, we end the proof of Theorem 5.2.

Remark 5.3. The equation in (3.1) is invariant under the inverse transformation, that is, if $u(t, x)$ solves (3.1) with initial data $u_{0}(x)$, so does $-u(t, x)$ with initial data $-u_{0}(x)$.

Thanks to Remarks 5.3, 5.1 and the proof of Theorem 5.2, we may readily derive the following wave-breaking result.

Theorem 5.3. Let $m_{0} \in H^{s}(\mathbb{R}), s>1 / 2$ and $T>0$ be the maximal time of existence of the corresponding solution $m(t, x)$ to (3.1) with the initial data $m_{0}$. Assume that $m_{0}(x)=$ $\left(1-\partial_{x}^{2}\right) u_{0} \leq 0$ for all $x \in \mathbb{R}$, and $m_{0}\left(x_{0}\right)<0$ for some $x_{0} \in \mathbb{R}$, and

$$
\partial_{x} u_{0}\left(x_{0}\right)>\left(\frac{\sqrt{2}\left\|u_{0}\right\|_{H^{1}}^{3}}{-m_{0}\left(x_{0}\right)}\right)^{\frac{1}{2}}
$$

Then the solution $m(t, x)$ blows up at some time $T_{0}$ with

$$
T_{0} \leq t^{*}:=\frac{\partial_{x} u_{0}\left(x_{0}\right)}{\sqrt{2}\left\|u_{0}\right\|_{H^{1}}^{3}}-\frac{1}{2} \sqrt{\left(\frac{\sqrt{2} \partial_{x} u_{0}\left(x_{0}\right)}{\left\|u_{0}\right\|_{H^{1}}^{3}}\right)^{2}+\frac{2 \sqrt{2}}{\left\|u_{0}\right\|_{H^{1}}^{3} m_{0}\left(x_{0}\right)}} .
$$

Moreover when $T_{0}=t^{*}$, the following estimate of the blow-up rate holds

$$
\lim \inf _{t \rightarrow T_{0}^{-}}\left(\left(T_{0}-t\right) \inf _{x \in \mathbb{R}} M(t, x)\right) \leq-1 .
$$

Remark 5.4. It is well known $[10]$ that if the initial data $u_{0} \in H^{3}(\mathbb{R})$ and $m_{0}=\left(1-\partial_{x}^{2}\right) u_{0}$ does not change sign, then the corresponding solution to the CH equation exists globally. While Theorem 5.2 and Remark 5.3 show that, even if the initial potential $m_{0}$ does not change sign, equation (3.1) may blow-up in finite time.

\section{Peaked solutions in The CASE $\gamma=0$}

In this section, we discuss the existence of single and multi-peakon solutions to the modified $\mathrm{CH}$ equation (1.1) with $\gamma=0$. Recall first that the single peakon of the $\mathrm{CH}$ equation (1.5) with $\gamma=0$ is given by

$$
u(t, x)=c e^{-|x-c t|}, \quad c \in \mathbb{R} .
$$

Multi-peakon solutions have the form

$$
u(t, x)=\sum_{i=1}^{N} p_{i}(t) e^{-\left|x-q_{i}(t)\right|},
$$


where $p_{i}(t)$ and $q_{i}(t), i=1,2, \cdots, N$ satisfy the following Hamiltonian system of ordinary differential equations:

$$
\begin{aligned}
& q_{i}^{\prime}(t)=\sum_{j=1}^{N} p_{j} e^{-\left|q_{i}-q_{j}\right|}, \quad i=1,2, \cdots, N, \\
& p_{i}^{\prime}(t)=\sum_{j=1}^{N} p_{i} p_{j} \operatorname{sign}\left(q_{i}-q_{j}\right) e^{-\left|q_{i}-q_{j}\right|},
\end{aligned}
$$

which has the canonical symplectic structure and Hamiltonian function

$$
H=\frac{1}{2} \sum_{i, j=1}^{N} p_{i}(t) p_{j}(t) e^{-\left|q_{i}(t)-q_{j}(t)\right|} .
$$

A rigorous analysis of the Hamiltonian system (6.2) can be found in [30]. It is worth mentioning that the Novikov equation also admits multi-peakons [34, 35]. The amplitudes of the multi-peakons of both the $\mathrm{CH}$ equation and the Novikov equation depend on time. Interestingly, as we now show, the amplitudes of multi-peakons for (3.1) with $\gamma=0$ are independent of time.

Theorem 6.1. For any $a \neq 0$, the peaked functions of the form

$$
u_{a}(t, x)=a e^{-|x-c t|}, \text { where } c=\frac{2}{3} a^{2},
$$

is a global weak solution to (3.1) with $\gamma=0$, in the sense of Definition 3.2.

Remark 6.1. Note that all peakons (6.4) move with positive wave speed, $c>0$. Each positive wave speed has a peakon and anti-peakon of opposite amplitudes: $a= \pm \sqrt{3 c / 2}$.

Remark 6.2. At each time $t \geq 0$, the peaked solutions $u_{a}(t, \cdot)$ belong to the Lipschitz space $W^{1, \infty}(\mathbb{R})$. However, we do not know whether general initial data in the space $W^{1, \infty}(\mathbb{R})$ produces a global weak solution. It's also not clear whether there is a global weak solution belonging to the space $W_{\text {loc }}^{1,3}(\mathbb{R})$ but not in $W^{1, \infty}(\mathbb{R})$, even for special initial data. Resolving these questions is a goal of our future work.

Proof of Theorem 6.1. We first claim that, for all $t \in \mathbb{R}^{+}$,

$$
\partial_{x} u_{a}(t, x)=-\operatorname{sign}(x-c t) u_{a}(t, x)
$$

in the sense of distribution $\mathcal{S}^{\prime}(\mathbb{R})$. Clearly (6.5) belongs to $L^{\infty}(\mathbb{R})$. Moreover, for any test function $\varphi(\cdot) \in C_{c}^{\infty}(\mathbb{R})$, using integration by parts,

$$
\begin{aligned}
\int_{\mathbb{R}} \operatorname{sign}(y) e^{-|y|} \varphi(y) d y=-\int_{-\infty}^{0} e^{y} \varphi(y) d y+\int_{0}^{+\infty} e^{-y} \varphi(y) d y \\
=-\varphi(0)+\int_{-\infty}^{0} e^{y} \varphi^{\prime}(y) d y+\varphi(0)+\int_{0}^{+\infty} e^{-y} \varphi^{\prime}(y) d y=\int_{\mathbb{R}} e^{-|y|} \varphi^{\prime}(y) d y
\end{aligned}
$$

which proves the claim.

Let us now set $u_{0, c}(x):=u_{a}(0, x)$ for $x \in \mathbb{R}$. Then

$$
\lim _{t \rightarrow 0^{+}}\left\|u_{a}(t, \cdot)-u_{0, c}(\cdot)\right\|_{W^{1, \infty}}=0 .
$$

As in (6.5), we have

$$
\partial_{t} u_{a}(t, x)=c \operatorname{sign}(x-c t) u_{a}(t, x) \in L^{\infty}(\mathbb{R}) \text { for all } t \geq 0 .
$$


Hence, using (6.6), (6.5), (6.7), and integration by parts, we deduce from that, for every test function $\varphi(t, x) \in C_{c}^{\infty}([0,+\infty) \times \mathbb{R})$,

$$
\begin{gathered}
\int_{0}^{+\infty} \int_{\mathbb{R}}\left(u_{a} \partial_{t} \varphi+\frac{1}{3} u_{a}^{3} \partial_{x} \varphi+\frac{1}{3}\left(\partial_{x} u_{a}\right)^{3} \varphi\right) d x d t+\int_{\mathbb{R}} u_{a}(0, x) \varphi(0, x) d x \\
=-\int_{0}^{+\infty} \int_{\mathbb{R}} \varphi\left(\partial_{t} u_{a}+u_{a}^{2} \partial_{x} u_{a}-\frac{1}{3}\left(\partial_{x} u_{a}\right)^{3}\right) d x d t \\
=-\int_{0}^{+\infty} \int_{\mathbb{R}} \varphi \operatorname{sign}(x-c t) u_{a}\left(c-\frac{2}{3} u_{a}^{2}\right) d x d t
\end{gathered}
$$

On the other hand, using (3.3),

$$
\begin{aligned}
\int_{0}^{+\infty} & \int_{\mathbb{R}}\left[\left(1-\partial_{x}^{2}\right)^{-1}\left(\frac{2}{3} u_{a}^{3}+u_{a}\left(\partial_{x} u_{a}\right)^{2}\right) \partial_{x} \varphi-\frac{1}{3}\left(1-\partial_{x}^{2}\right)^{-1}\left(\partial_{x} u_{a}\right)^{3} \varphi\right] d x d t \\
& =-\int_{0}^{+\infty} \int_{\mathbb{R}}\left[\varphi \partial_{x} p *\left(u_{a}\left(\partial_{x} u_{a}\right)^{2}\right)+\varphi p *\left(2 u_{a}^{2} \partial_{x} u_{a}+\frac{1}{3}\left(\partial_{x} u_{a}\right)^{3}\right)\right] d x d t .
\end{aligned}
$$

We calculate from (6.5) that

$$
2 u_{a}^{2} \partial_{x} u_{a}+\frac{1}{3}\left(\partial_{x} u_{a}\right)^{3}=-2 \operatorname{sign}(x-c t) u_{a}^{3}-\frac{1}{3}(\operatorname{sign}(x-c t))^{3} u_{a}^{3}=\frac{7}{9} \partial_{x}\left(u_{a}^{3}\right),
$$

which together with (6.9) leads to

$$
\begin{gathered}
\int_{0}^{+\infty} \int_{\mathbb{R}}\left[\left(1-\partial_{x}^{2}\right)^{-1}\left(\frac{2}{3} u_{a}^{3}+u_{a}\left(\partial_{x} u_{a}\right)^{2}\right) \partial_{x} \varphi-\frac{1}{3}\left(1-\partial_{x}^{2}\right)^{-1}\left(\partial_{x} u_{a}\right)^{3} \varphi\right] d x d t \\
=-\int_{0}^{T} \int_{\mathbb{R}} \varphi \cdot \partial_{x} p *\left(u_{a}\left(\partial_{x} u_{a}\right)^{2}+\frac{7}{9} u_{a}^{3}\right) d x d t
\end{gathered}
$$

Notice from (6.5) that $\partial_{x} p(x)=-\frac{1}{2} \operatorname{sign}(x) e^{-|x|}$ for $x \in \mathbb{R}$, we have

$$
\begin{array}{rl}
\partial_{x} p & *\left(u_{a}\left(\partial_{x} u_{a}\right)^{2}+\frac{7}{9} u_{a}^{3}\right)(t, x) \\
& =-\frac{1}{2} \int_{-\infty}^{+\infty} \operatorname{sign}(x-y) e^{-|x-y|}\left(\frac{7}{9}+\operatorname{sign}^{2}(y-c t)\right) \frac{3 c}{2} \sqrt{\frac{3 c}{2}} e^{-3|y-c t|} d y d t .
\end{array}
$$

When $x>c t$, we split the right hand side of (6.11) into the following three parts:

$$
\begin{aligned}
& \partial_{x} p *\left(u_{a}\left(\partial_{x} u_{a}\right)^{2}+\frac{7}{9} u_{a}^{3}\right)(t, x) \\
& =-\frac{3 c}{4} \sqrt{\frac{3 c}{2}}\left(\int_{-\infty}^{c t}+\int_{c t}^{x}+\int_{x}^{+\infty}\right) \operatorname{sign}(x-y) e^{-|x-y|}\left(\frac{7}{9}+\operatorname{sign}^{2}(y-c t)\right) e^{-3|y-c t|} d y \\
& =: I_{1}+I_{2}+I_{3} .
\end{aligned}
$$

We directly compute $I_{1}$ as follows:

$$
\begin{aligned}
I_{1} & =-\frac{3 c}{4} \sqrt{\frac{3 c}{2}} \int_{-\infty}^{c t} \frac{16}{9} e^{-(x-y)} e^{3(y-c t)} d y \\
& =-\frac{4 c}{3} \sqrt{\frac{3 c}{2}} e^{-(x+3 c t)} \int_{-\infty}^{c t} e^{4 y} d y=-\frac{c}{3} \sqrt{\frac{3 c}{2}} e^{c t-x} .
\end{aligned}
$$


In a similar manner,

$$
\begin{aligned}
I_{2} & =-\frac{3 c}{4} \sqrt{\frac{3 c}{2}} \int_{c t}^{x} \frac{16}{9} e^{-(x-y)} e^{-3(y-c t)} d y \\
& =-\frac{4 c}{3} \sqrt{\frac{3 c}{2}} e^{-(x-3 c t)} \int_{c t}^{x} e^{-2 y} d y=-\frac{2 c}{3} \sqrt{\frac{3 c}{2}}\left(e^{c t-x}-e^{3(c t-x)}\right) .
\end{aligned}
$$

and

$$
\begin{aligned}
I_{3} & =\frac{3 c}{4} \sqrt{\frac{3 c}{2}} \int_{x}^{+\infty} \frac{16}{9} e^{x-y} e^{-3(y-c t)} d y \\
& =\frac{4 c}{3} \sqrt{\frac{3 c}{2}} e^{(x+3 c t)} \int_{x}^{+\infty} e^{-4 y} d y=\frac{c}{3} \sqrt{\frac{3 c}{2}} e^{3(c t-x)} .
\end{aligned}
$$

Plugging (6.13)-(6.15) into (6.12), we deduce that for $x>c t$

$$
\partial_{x} p *\left(u_{a}\left(\partial_{x} u_{a}\right)^{2}+\frac{7}{9} u_{a}^{3}\right)(t, x)=c \sqrt{\frac{3 c}{2}}\left(e^{3(c t-x)}-e^{c t-x}\right) .
$$

While for the case $x \leq c t$, we split the right hand side of (6.11) into the following three parts:

$$
\begin{aligned}
& \partial_{x} p *\left(u_{a}\left(\partial_{x} u_{a}\right)^{2}+\frac{7}{9} u_{a}^{3}\right)(t, x) \\
& =-\frac{3 c}{4} \sqrt{\frac{3 c}{2}}\left(\int_{-\infty}^{x}+\int_{x}^{c t}+\int_{c t}^{+\infty}\right) \operatorname{sign}(x-y) e^{-|x-y|}\left(\frac{7}{9}+\operatorname{sign}^{2}(y-c t)\right) e^{-3|y-c t|} d y \\
& =: I I_{1}+I I_{2}+I I_{3} .
\end{aligned}
$$

For $I I_{1}$, a direct computation gives rise to

$$
\begin{aligned}
I I_{1} & =-\frac{3 c}{4} \sqrt{\frac{3 c}{2}} \int_{-\infty}^{x} \frac{16}{9} e^{-(x-y)} e^{3(y-c t)} d y \\
& =-\frac{4 c}{3} \sqrt{\frac{3 c}{2}} e^{-(x+3 c t)} \int_{-\infty}^{x} e^{4 y} d y=-\frac{c}{3} \sqrt{\frac{3 c}{2}} e^{3(x-c t)} .
\end{aligned}
$$

Similarly, one obtains

$$
\begin{aligned}
I I_{2} & =\frac{3 c}{4} \sqrt{\frac{3 c}{2}} \int_{x}^{c t} \frac{16}{9} e^{x-y} e^{3(y-c t)} d y \\
& =\frac{4 c}{3} \sqrt{\frac{3 c}{2}} e^{x-3 c t} \int_{x}^{c t} e^{2 y} d y=\frac{2 c}{3} \sqrt{\frac{3 c}{2}}\left(e^{x-c t}-e^{3(x-c t)}\right) .
\end{aligned}
$$

and

$$
\begin{aligned}
I I_{3} & =\frac{3 c}{4} \sqrt{\frac{3 c}{2}} \int_{c t}^{+\infty} \frac{16}{9} e^{x-y} e^{-3(y-c t)} d y \\
& =\frac{4 c}{3} \sqrt{\frac{3 c}{2}} e^{(x+3 c t)} \int_{c t}^{+\infty} e^{-4 y} d y=\frac{c}{3} \sqrt{\frac{3 c}{2}} e^{x-c t} .
\end{aligned}
$$

Plugging (6.18)-(6.20) into (6.7), we deduce that for $x \leq c t$

$$
\partial_{x} p *\left(u_{a}\left(\partial_{x} u_{a}\right)^{2}+\frac{7}{9} u_{a}^{3}\right)(t, x)=-c \sqrt{\frac{3 c}{2}}\left(e^{3(x-c t)}-e^{x-c t}\right) .
$$


On the other hand, using the definition of $u_{a}$,

$$
\operatorname{sign}(x-c t) u_{a}\left(c-\frac{2}{3} u_{a}^{2}\right)(t, x)=\left\{\begin{array}{l}
-c \sqrt{\frac{3 c}{2}}\left(e^{3(c t-x)}-e^{c t-x}\right), \quad \text { for } \quad x>c t, \\
c \sqrt{\frac{3 c}{2}}\left(e^{3(x-c t)}-e^{x-c t}\right), \quad \text { for } \quad x \leq c t
\end{array}\right.
$$

which along with (6.16) and (6.21) yields

$$
\partial_{x} p *\left[u_{a}\left(\partial_{x} u_{a}\right)^{2}+\frac{7}{9} u_{a}^{3}\right](t, x)+\operatorname{sign}(x-c t)\left[u_{a}\left(c-\frac{2}{3} u_{a}^{2}\right)\right](t, x)=0,
$$

for all $(t, x) \in \mathbb{R}^{+} \times \mathbb{R}$. Therefore, in view of (6.8), (6.11) and (6.22), we conclude that

$$
\begin{aligned}
\int_{0}^{+\infty} \int_{\mathbb{R}}\left(u_{a} \partial_{t} \varphi\right. & +\frac{1}{3} u_{a}^{3} \partial_{x} \varphi+\frac{1}{3}\left(\partial_{x} u_{a}\right)^{3} \varphi+\left(1-\partial_{x}^{2}\right)^{-1}\left(\frac{2}{3} u_{a}^{3}+u\left(\partial_{x} u_{a}\right)^{2}\right) \partial_{x} \varphi \\
& \left.-\frac{1}{3}\left(1-\partial_{x}^{2}\right)^{-1}\left(\partial_{x} u_{a}\right)^{3} \varphi\right) d x d t+\int_{\mathbb{R}} u_{a}(0, x) \varphi(0, x) d x=0
\end{aligned}
$$

for every test function $\varphi(t, x) \in C_{c}^{\infty}([0,+\infty) \times \mathbb{R})$, which completes the proof of Theorem 6.1 .

We now derive the multipeakon solutions of equation (3.1). Assume that equation (3.1) with $\gamma=0$ has an $N$-peakon solution of the form (6.1). It follows from Definition 3.2 that for any $\varphi(t, x) \in C_{c}^{\infty}([0, \infty) \times \mathbb{R})$, the solution (6.1) satisfies

$$
\int_{0}^{\infty} \int_{\mathbb{R}}\left[u_{t}+\left(u^{2}-\frac{1}{3} u_{x}^{2}\right) u_{x}+\left(1-\partial_{x}^{2}\right)^{-1} \partial_{x}\left(\frac{2}{3} u^{3}+u u_{x}^{2}\right)+\frac{1}{3}\left(1-\partial_{x}^{2}\right)^{-1} u_{x}^{3}\right] \varphi(x) d x d t=0,
$$

which is equivalent to the equation

$$
\int_{0}^{\infty} \int_{\mathbb{R}}\left[u_{t}\left(\psi-\psi_{x x}\right)+\frac{1}{3}\left(u^{3} \psi_{x x x}+u_{x}^{3} \psi_{x x}\right)-u\left(u^{2}+u_{x}^{2}\right) \psi_{x}\right] d x d t=0,
$$

where $\varphi=\psi-\psi_{x x}, \psi(x) \in C_{c}^{\infty}([0, \infty) \times \mathbb{R})$.

A straightforward computation gives

$$
\begin{aligned}
& I_{1}=\int_{0}^{\infty} \int_{\mathbb{R}} u_{t}\left(\psi-\psi_{x x}\right) d x d t \\
& =\sum_{j=1}^{N} \int_{0}^{\infty} \int_{-\infty}^{q_{j}(t)}\left(p_{j}^{\prime}-p_{j} q_{j}^{\prime}\right) e^{x-q_{j}}\left(\psi-\psi_{x x}\right) d x d t \\
& +\sum_{j=1}^{N} \int_{0}^{\infty} \int_{q_{j}}^{\infty}\left(p_{j}^{\prime}+p_{j} q_{j}^{\prime}\right) e^{-\left(x-q_{j}\right)}\left(\psi-\psi_{x x}\right) d x d t \\
& =2 \int_{0}^{\infty} \sum_{j=1}^{N}\left(p_{j}^{\prime} \psi\left(q_{j}\right)+p_{j} q_{j}^{\prime} \psi_{x}\left(q_{j}\right)\right) d t, \\
& I_{2}=\int_{0}^{\infty} \int_{\mathbb{R}}\left[\frac{1}{3}\left(u^{3} \psi_{x x x}+u_{x}^{3} \psi_{x x}\right)-u\left(u^{2}+u_{x}^{2}\right) \psi_{x}\right] d x d t \\
& =\int_{0}^{\infty}\left(\int_{-\infty}^{q_{1}}+\sum_{j=1}^{N-1} \int_{q_{j}}^{q_{j+1}}+\int_{q_{N}}^{\infty}\right)\left[\frac{1}{3}\left(u^{3} \psi_{x x x}+u_{x}^{3} \psi_{x x}\right)-u\left(u^{2}+u_{x}^{2}\right) \psi_{x}\right] d x d t \\
& \left.=2 \int_{0}^{\infty} \sum_{j=1}^{N} p_{j}\left(-\frac{2}{3} p_{j}^{3}-2 \sum_{i=1}^{N} p_{j} p_{i} e^{-\left|q_{i}-q_{j}\right|}-4 \sum_{1 \leq k<j, j<i \leq N} p_{k} p_{i} e^{-\left|q_{k}-q_{i}\right|}\right)\right) \psi_{x}\left(q_{j}\right) d t .
\end{aligned}
$$


Substituting the preceding expressions into (6.23), we obtain the following system

$$
\begin{aligned}
p_{j}^{\prime} & =0 \\
q_{j}^{\prime} & =\frac{2}{3} p_{j}^{2}+2 \sum_{i=1}^{N} p_{j} p_{i} e^{-\left|q_{i}-q_{j}\right|}+4 \sum_{1 \leq k<j, j<i \leq N} p_{k} p_{i} e^{-\left|q_{k}-q_{i}\right|} .
\end{aligned}
$$

For $N=2$, the system (6.24) can be easily integrated. In this case, without loss of generality, assume $q_{1}(t)<q_{2}(t)$, we obtain the general solution of system (6.24) given by

$$
\begin{aligned}
& p_{1}=\sqrt{\frac{3}{2} c_{1}}, \quad p_{2}=\sqrt{\frac{3}{2} c_{2},} \\
& q_{1}=c_{1} t+\frac{3 \sqrt{c_{1} c_{2}}}{c_{1}-c_{2}} e^{\left(c_{1}-c_{2}\right)\left(t-t_{0}\right)}+x_{0}, \\
& q_{2}=c_{2} t+\frac{3 \sqrt{c_{1} c_{2}}}{c_{1}-c_{2}} e^{\left(c_{1}-c_{2}\right)\left(t-t_{0}\right)}+x_{0}+\left(c_{2}-c_{1}\right) t_{0},
\end{aligned}
$$

where $c_{1}<c_{2}$, and $x_{0}$ and $t_{0}$ are integration constants, which describe translation invariance for $x$ and $t$. Setting $x_{0}=t_{0}=0$, we derive two-peakon solutions of the modified $\mathrm{CH}$ equation in the explicit form

$$
\begin{aligned}
u(t, x)=\sqrt{\frac{3}{2} c_{1}} & \exp \left\{-\left|x-c_{1} t-\frac{3 \sqrt{c_{1} c_{2}}}{c_{1}-c_{2}} e^{\left(c_{1}-c_{2}\right) t}\right|\right\} \\
& +\sqrt{\frac{3}{2} c_{2}} \exp \left\{-\left|x-c_{2} t-\frac{3 \sqrt{c_{1} c_{2}}}{c_{1}-c_{2}} e^{\left(c_{1}-c_{2}\right) t}\right|\right\} .
\end{aligned}
$$

As $t \rightarrow \infty$, the two peakons separate:

$$
u(t, x) \approx \sqrt{\frac{3}{2} c_{1}} e^{-\left|x-c_{1} t\right|}+\sqrt{\frac{3}{2} c_{2}} e^{-\left|x-c_{2} t\right|},
$$

whereas when $t \rightarrow-\infty$, the peakons separate and go off to $\infty$ at an exponentially increasing rate.

Representative profiles of a single peakon and a double peakon solution are plotted in Figures 1 and 2, respectively.

Acknowledgments. The authors would like to thank the referees for constructive suggestions and comments. The work of Gui is supported in part by the NSF of China under the grants 11001111 and 11171241, and Jiangsu University grants 10JDG141 and 10JDG157. The work of Liu is supported in part by NSF grant DMS-0906099 and NHARP grant 003599-0001-2009. The work of Olver is supported in part by NSF grant DMS-1108894. The work of Qu is supported in part by NSF-China for Distinguished Young Scholars grant10925104 and Ph.D. Programs Foundation of Ministry of Education of China-20106101110008.

\section{REFERENCES}

[1] M. S. Alber, R. Camassa, D. D. Holm, And J. E. Marsden, The geometry of peaked solitons and billiard solutions of a class of integrable PDE's, Lett. Math. Phys., 32 (1994), 137-151.

[2] H. Bahouri, J. Y. Chemin, And R. Danchin, Fourier Analysis and Nonlinear Partial Differential Equations. Grundlehren der mathematischen Wissenschaften 343, Springer-Verlag, Berlin Heidelberg, 2011.

[3] R. Beals, D. H. Sattinger and J. Szmigielski, Acoustic scattering and the extended Korteweg-de Vries hierarchy, Adv. Math., 140 (1998), 190-206.

[4] A. Boutet de Monvel, A. Kostenko, D. Shepelsky, And G. Teschl, Long-time asymptotics for the Camassa-Holm equation, SIAM J. Math. Anal., 41 (2009), 1559-1588.

[5] J. C. BRunelli, The short pulse hierarchy, J. Math. Phys., 46 (2005), 123507. 


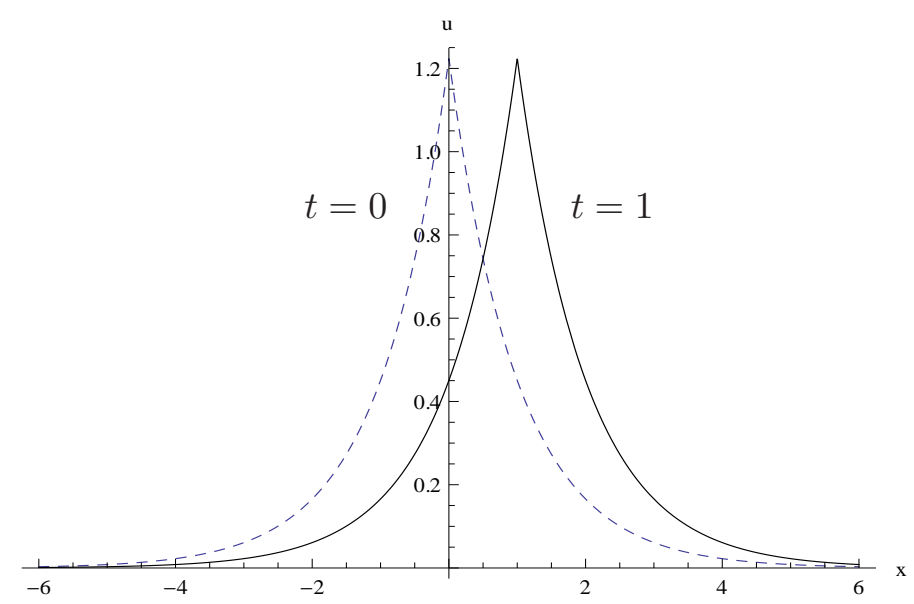

FIGURE 1. Single peakon solution of the modified $\mathrm{CH}$ equation with $\gamma=0$, wave speed $c=1$, and amplitude $a=\sqrt{3 / 2}$.

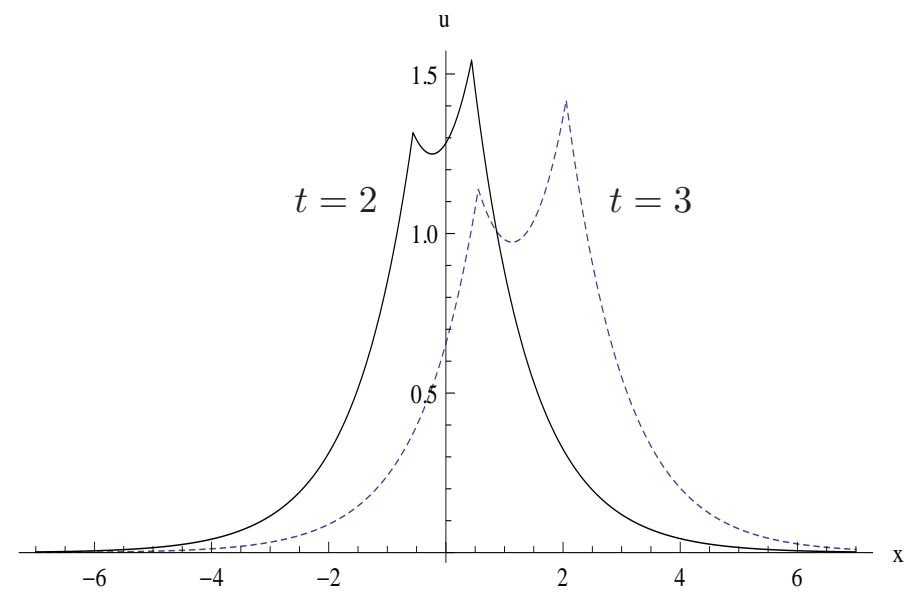

FigURE 2. Double peakon solution of the modified $\mathrm{CH}$ equation with $\gamma=0$, wave speeds $c_{1}=1 / 2, c_{2}=1$, and amplitudes $a_{1}=\sqrt{3 / 4}, a_{2}=\sqrt{3 / 2}$.

[6] R. CAmassa AND D. D. Holm, An integrable shallow water equation with peaked solitons, Phys. Rev. Lett., 71 (1993), 1661-1664.

[7] C. S. CaO, D. D. Holm, AND E. S. Titi, Traveling wave solutions for a class of onedimensional nonlinear shallow water wave models, J. Dynam. Differential Equations, 16 (2004), 167-178.

[8] K. S. ChOU AND C. Z. QU, Integrable equations arising from motions of plane curves I, Physica D, 162 (2002), 9-33.

[9] Y. Chung, C.K.R.T. Jones, T. SChÄFER, And C.E. WAYNE, Ultra-short pulses in linear and nonlinear media, Nonlinearity, 18 (2005), $1351-1374$.

[10] A. Constantin, Existence of permanent and breaking waves for a shallow water equation: a geometric approach, Ann. Inst. Fourier (Grenoble), 50 (2000), 321-362.

[11] A. CONSTANTIN AND J. EsCHER, Wave breaking for nonlinear nonlocal shallow water equations, Acta Math., 181 (1998), 229-243.

[12] A. CONSTANTIN AND J. EsCHER, On the blow-up rate and the blow-up set of breaking waves for a shallow water equation, Math. Z., 233 (2000), 75-91. 
[13] A. Constantin And J. Escher, Global existence and blow-up for a shallow water equation, Ann. Scuola Norm. Sup. Pisa, 26 (1998), 303-328.

[14] A. Constantin, V. S. Gerdjikov and R. I. IVAnov, Inverse scattering transform for the Camassa-Holm equation, Inverse Problems, 22 (2006), 2197-2207

[15] A. Constantin And R. I. Ivanov, On an integrable two-component Camassa-Holm shallow water system, Phys. Lett. A, 372 (2008), 7129-7132.

[16] A. Constantin, T. Kappeler, B. Kolev, And P. Topalov, On geodesic exponential maps of the Virasoro group, Ann. Glob. Anal. Geom., 31 (2007), 155-180.

[17] A. Constantin And D. LAnnes, The hydrodynamical relevance of the Camassa-Holm and Degasperis-Procesi equations, Arch. Ration. Mech. Anal., 192 (2009), 165-186.

[18] A. Constantin And H. P. McKean, A shallow water equation on the circle, Comm. Pure Appl. Math., 52 (1999), 949-982.

[19] M. FISHER AND J. SCHIFF, The Camassa Holm equation: conserved quantities and the initial value problem, Phys. Lett. A, 259 (1999), 371-376.

[20] A. S. Fokas, P. J. Olver, And P. RosenAU, A plethora of integrable bi-Hamiltonian equations, in: Algebraic Aspects of Integrable Systems: In Memory of Irene Dorfman, A.S. Fokas and I.M. Gel'fand, eds., Progress in Nonlinear Differential Equations, vol. 26, Birkhäuser, Boston, 1996, pp. 93-101.

[21] Y. FU, Y. LIU, AND C. Z. QU, Well-posedness and blow-up solution for a modified twocomponent periodic Camassa-Holm system with peakons, Math. Ann., 348 (2010), 415-448.

[22] Y. FU, G. GUI, Y. LiU, AND C. Z. QU, On the Cauchy problem for the integrable CamassaHolm type equation with cubic nonlinearity, Preprint, 2011.

[23] B. FUCHSSTEINER, Some tricks from the symmetry-toolbox for nonlinear equations: generalizations of the Camassa-Holm equation, Physica D, 95 (1996), 229-243.

[24] B. FUCHSSTEINER AND A. S. FOKAS, Symplectic structures, their Bäcklund transformations and hereditary symmetries, Physica D, 4 (1981/1982), 47-66.

[25] R. E. Goldstein And D. M. Petrich, The Korteweg-de Vries hierarchy as dynamics of closed curves in the plane, Phys. Rev. Lett., 67 (1991), 3203-3206.

[26] P. GórKa AND E. G. Reyes, The modified Camassa-Holm equation, Int. Math. Res. Notes, 2011 (2011), 2617-2649.

[27] G. GUi AND Y. LiU, On the global existence and wave-breaking criteria for the two-component Camassa-Holm system, J. Funct. Anal., 258 (2010), 4251-4278.

[28] H. W. GugGenheimer, Differential Geometry, McGraw-Hill, New York, 1963.

[29] H. Hasimoto, A soliton on a vortex filament, J. Fluid Mech., 51 (1972), 477-485.

[30] H. Holden AND X. RAYNAUd, A convergent numerical scheme for the Camassa-Holm equation based on multipeakons, Discrete Contin. Dyn. Syst. A., 14 (2006), 505-523.

[31] D. D. Holm And R. I. IVAnOV, Multi-component generalizations of the CH equation: geometrical aspects, peakons and numerical examples, J. Phys. A, 43 (2010), 492001.

[32] D. D. Holm And R. I. Ivanov, Two-component CH system: inverse scattering, peakons and geometry, Inverse Problems, 27 (2011), 045013.

[33] D. D. Holm, L. Ó NÁraigh, C. TronCI, Singular solutions of a modified two-component Camassa-Holm equation, Physical Review E, 79 (2009), 016601.

[34] A. N. Hone, H. Lundmark, And J. Szmigielski, Explicit multipeakon solutions of Novikov's cubically nonlinear integrable Camassa-Holm type equation, Dyn. Partial Diff. Eq., 6 (2009), 253-289.

[35] A. N. Hone AND J. P. WANG, Integrable peakon equations with cubic nonlinearity, J. Phys. A, 41 (2008), 372002

[36] R. S. Johnson, Camassa-Holm, Korteweg-de Vries and related models for water waves, $J$. Fluid Mech., 455 (2002), 63-82.

[37] S. KouranbaEVA, The Camassa-Holm equation as a geodesic flow on the diffeomorphism group, J. Math. Phys., 40 (1999), 857-868.

[38] J. Langer and R. Perline, Poisson geometry of the filament equation, J. Nonlin. Sci., 1 (1991), 71-93.

[39] Y. A. Li AND P. J. OlvER, Convergence of solitary-wave solutions in a perturbed biHamiltonian dynamical system. I. Compactons and peakons, Discrete Cont. Dyn. Syst., 4 (1998), 419-432.

[40] Y. A. Li AND P. J. OLVER, Well-posedness and blow-up solutions for an integrable nonlinearly dispersive model wave equation, J. Diff. Eq., 162 (2000), 27-63. 
[41] Y. A. Li AND P. J. Olver, Convergence of solitary-wave solutions in a perturbed biHamiltonian dynamical system. II. Complex analytic behavior and convergence to non-analytic solutions, Discrete Cont. Dyn. Syst., 4 (1998), 159-191.

[42] Y. A. Li, P. J. Olver, AND P. Rosenau, Non-analytic solutions of nonlinear wave models, in: Nonlinear Theory of Generalized Functions, M. Grosser, G. Hörmann, M. Kunzinger, and M. Oberguggenberger, eds., Research Notes in Mathematics, vol. 401, Chapman and Hall/CRC, New York, 1999, pp. 129-145.

[43] Y. LiU, D. Pelinovsky, And A. SAKovich, Wave breaking in the short-pulse equation, Dynamics of PDE, 6 (2009), 291-310.

[44] G. MisioleK, A shallow water equation as a geodesic flow on the Bott-Virasoro group, $J$. Geom. Phys., 24 (1998), 203-208.

[45] V. Novikov, Generalizations of the Camassa-Holm equation, J. Phys. A, 42 (2009), 342002.

[46] P. J. Olver, Applications of Lie Groups to Differential Equations, Second Edition, Graduate Texts in Mathematics, vol. 107, Springer-Verlag, New York, 1993.

[47] P. J. Olver, Invariant submanifold flows, J. Phys. A, 41 (2008) 344017.

[48] P. J. Olver And P. Rosenau, Tri-Hamiltonian duality between solitons and solitary-wave solutions having compact support, Phys. Rev. E, 53 (1996), 1900-1906.

[49] U. PinkalL, Hamiltonian flows on the space of star-shaped curves, Results Math., 27 (1995), 328-332.

[50] Z. QIAO, A new integrable equation with cuspons and W/M-shape-peaks solitons, J. Math. Phys., 47 (2006), 112701.

[51] Z. QiaO AND X. Q. LI, An integrable equation with nonsmooth solitons, Theor. Math. Phys., 267 (2011), 584-589.

[52] E. G. Reyes, Geometric integrability of the Camassa-Holm equation, Lett. Math. Phys., 59 (2002), 117-131.

[53] A. SAKOVich And S. SAKOVICH, Solitary wave solutions of the short pulse equation, J. Phys. A, 39 (2006), L361-L367.

[54] T. SCHÄFER AND C. E. WAYNE, Propagation of ultra-short optical pulses in cubic nonlinear media, Physica D, 196 (2004), 90-105.

[55] J. SCHIFF, The Camassa-Holm equation: a loop group approach, Physica D, 121 (1998), 2443.

[56] F. TIĞLAY, The periodic Cauchy problem of the modified Hunter-Saxton equation, J. Evol. Eq., 5 (2005), 509-527.

[57] F. TIĞLAY, The periodic Cauchy problem for Novikov's equation, Int. Math. Res. Notes, 2011 (2011), 4633-4648.

[58] M. WAdati, K. Konno, And Y. ICHiKaWA, New integrable nonlinear evolution equations, J. Phys. Soc. Japan, 47 (1979), 1698-1700.

(Guilong Gui) Department of Mathematics, Jiangsu University, Zhenjiang 212013, P. R. China, And The Institute of Mathematical Sciences, The Chinese University of Hong Kong, Shatin, N.T., HONG Kong

E-mail address: glgui@amss.ac.cn

(Yue Liu) Department of Mathematics, University of TeXas, Arlington, TX 76019-0408, USA

E-mail address: yliu@uta.edu USA

(Peter J. Olver) School of Mathematics, University of Minnesota, Minneapolis, MN 55455,

E-mail address: olver@math.umn.edu

(Changzheng Qu) Department of Mathematics, Ningbo University, Ningbo 315211, P.R. China

E-mail address: quchangzheng@nbu.edu.cn 